\title{
SU(5) D-brane realizations, Yukawa couplings and proton stability
}

\author{
P. Anastasopoulos, ${ }^{a}$ G.K. Leontaris, ${ }^{b}$ R. Richter $^{c}$ and A.N. Schellekens ${ }^{d, e, f}$ \\ a Technische Univ. Wien Inst. für Theoretische Physik, \\ A-1040 Vienna, Austria \\ ${ }^{b}$ Theoretical Physics Division, Ioannina University, \\ GR-45110 Ioannina, Greece \\ ${ }^{c}$ I.N.F.N. - Sezione di Roma "Tor Vergata", \\ 00133, Roma, Italy \\ ${ }^{d}$ NIKHEF, \\ Science Park 105, 1098 XG Amsterdam, The Netherlands \\ e IMAPP, Radboud Universiteit Nijmegen, \\ The Netherlands \\ ${ }^{f}$ Instituto de Física Fundamental, CSIC, \\ Madrid, Spain \\ E-mail: pascal@hep.itp.tuwien.ac.at, leonta@uoi.gr, \\ rrichter@roma2.infn.it, t58@nikhef.nl
}

Abstract: We discuss SU(5) Grand Unified Theories in the context of orientifold compactifications. Specifically, we investigate two and three D-brane stack realizations of the Georgi-Glashow and the flipped SU(5) model and analyze them with respect to their Yukawa couplings. As pointed out in [1] the most economical Georgi-Glashow realization based on two stacks generically suffers from a disastrous large proton decay rate. We show that allowing for an additional U(1) D-brane stack this as well as other phenomenological problems can be resolved. We exemplify with globally consistent Georgi-Glashow models based on RCFT that these D-brane quivers can be indeed embedded in a global setting. These globally consistent realizations admit rigid $\mathrm{O}(1)$ instantons inducing the perturbatively missing coupling $\mathbf{1 0 1 0 5}^{\mathrm{H}}$. Finally we show that flipped SU(5) D-brane realizations even with multiple U(1) D-brane stacks are plagued by severe phenomenological drawbacks which generically cannot be overcome.

KEYWORDs: Intersecting branes models, D-branes, Superstring Vacua

ARXIV EPRINT: 1010.5188 


\section{Contents}

1 Introduction 1

2 Georgi-Glashow model $\quad 3$

2.1 D-brane realization 4

2.1.1 Three-stack quiver with perturbatively realized $\mu$-term 6

$\begin{array}{lll}2.1 .2 & \text { Three-stack quiver with non-perturbative } \mu \text {-term } & 7\end{array}$

$\begin{array}{ll}2.2 \text { Global realization of a three-stack quiver } & 8\end{array}$

3 Flipped SU(5) model 12

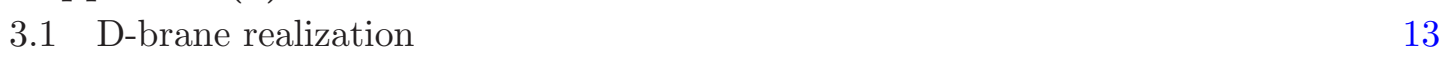

4 Conclusions $\quad 16$

A String consistency conditions and phenomenological constraints $\quad 17$

$\begin{array}{ll}\text { A.1 Tadpole cancellation } & 17\end{array}$

$\begin{array}{lll}\text { A.2 Massless U(1)'s } & 18\end{array}$

$\begin{array}{ll}\text { A.3 Derivation for RCFT models } & 19\end{array}$

$\begin{array}{ll}\text { A.4 Phenomenological requirements } & 21\end{array}$

B Georgi-Glashow realizations based on three stacks $\quad 21$

C Globally consistent 3-stack model $\quad 22$

$\begin{array}{lll}\text { C.1 Gepner orientifold of type I } & 23\end{array}$

C.2 Gepner orientifold of type II 24

\section{Introduction}

D-brane compactifications have been proven to be a promising framework for realistic string model building. The basic building blocks of such constructions are D-branes which fill out the four-dimensional space-time and wrap submanifolds in the internal manifold. The gauge bosons live on the world volume of the respective D-brane while chiral matter appears at intersections of different stacks of D-branes. The multiplicity of the latter is given by the number of intersections of the respective submanifolds in the internal space. Over the last decade many globally consistent semi-realistic D-brane models have been constructed (for recent reviews, see [2-4]) .

In this work we are investigating how supersymmetric SU(5) GUT's can be realized in this framework with the emphasis on the realization of the superpotential. ${ }^{1}$ The SU(5)

\footnotetext{
${ }^{1}$ For global supersymmetric SU(5) D-brane realizations, see [5-13]. For a related study of GUT's within this framework, see [14].
} 
gauge symmetry arises from a stack $a$ of 5 D-branes giving rise to the gauge symmetry $\mathrm{U}(5)_{a}$ which further splits into $\mathrm{U}(5)_{a}=\mathrm{SU}(5) \times \mathrm{U}(1)_{a}$. Here the abelian part generically acquires a mass via the Green-Schwarz mechanism and survives only as global symmetry. The $\mathbf{1 0}$ is localized at intersections of the U(5) stack $a$ and its orientifold image $a^{\prime}$. To accommodate for the other matter fields $\overline{\mathbf{5}}$, as well as the Higgs pair $\mathbf{5}^{\mathbf{H}}$ and $\overline{\mathbf{5}}^{\mathbf{H}}$, one needs the presence of at least one, but potentially more $\mathrm{U}(1)$ stacks. The $\overline{\mathbf{5}}, \mathbf{5}^{\mathbf{H}}$ and $\overline{\mathbf{5}}^{\mathbf{H}}$ appear then as bi-fundamentals at intersections of the U(5) stack and one of the U(1) stacks. The singlets under the $\mathrm{SU}(5)$ arise at intersections between different U(1)-stacks.

The perturbative superpotential is given by all gauge invariant couplings that are uncharged under all the global U(1)'s, the remnants of the Green-Schwarz mechanism. While the couplings

$$
10 \overline{55}^{\mathrm{H}} \quad \overline{5}^{\mathrm{H}} \mathbf{1} \quad \overline{5}^{\mathrm{H}} 5^{\mathrm{H}}
$$

can in principle be perturbatively realized the other desired coupling

$$
10_{(2,0)} \mathbf{1 0}_{(2,0)} \mathbf{5}_{(1,1)}^{\mathbf{H}}
$$

is perturbatively forbidden since it violates the global selection rules. Here the subscripts denote the charge of the respective matter fields under the global U(1)'s namely the one originating from the $\mathrm{U}(5)_{a}$ and the $\mathrm{U}(1)$ under which the Higgs field $\mathbf{5}^{\mathbf{H}}$ is charged. Obviously, this coupling is not neutral under these two global U(1)'s and therefore perturbatively forbidden.

Recently, it has been realized that D-instantons carry charge under these global U(1)'s [1517] (for recent reviews, see $[18,19]$ ). For a specific product of matter fields they can compensate for the overshoot in the global $\mathrm{U}(1)$ charge and induce the perturbatively missing couplings. For a rigid $O(1)$ instanton, which satisfies the severe constraints on the uncharged zero mode structure [20-23], the charge under the global $\mathrm{U}(1)_{x}$ arising from a stack of $N_{x}$ branes wrapping the cycle $\pi_{X}$ in the internal manifold is given by ${ }^{2}$

$$
Q_{x}=-N_{x} \pi_{E} \circ \pi_{x}
$$

Here $\pi_{E}$ denotes the orientifold invariant cycle wrapped by the D-instanton. The nonperturbative generation of Yukawa couplings via a rigid $O(1)$ instanton has been explicitly discussed in [33].

In this work we extend the analysis of supersymmetric SU(5) GUT's in a global context performed in [1], in which the authors pointed out that the most economical realization based on two stacks of D-branes poses severe phenomenological drawbacks. We investigate whether these drawbacks can be resolved by allowing additional D-brane stacks and find that the Georgi-Glashow model can be accommodated via three D-brane stacks, overcoming all problems encountered in the two D-brane stack realization. We present a global realization based on rational conformal field theory (RCFT), that admits a rigid $O(1)$

\footnotetext{
${ }^{2}$ Other instanton configurations, such as multi-instantons [24-27] and so called rigid U(1) instantons [25, 28-32] can induce superpotential terms.
} 


\begin{tabular}{|c|c|c|}
\hline Representation & SM matter embedding & Multiplicity \\
\hline $\mathbf{1 0}$ & $\left(q_{L}, u_{R}, e_{R}\right)$ & 3 \\
\hline$\overline{\mathbf{5}}$ & $\left(L, d_{R}\right)$ & 3 \\
\hline $\mathbf{1}$ & $\nu_{R}$ & 3 \\
\hline $\mathbf{5}_{\mathbf{H}}+\overline{\mathbf{5}}_{\mathbf{H}}$ & $\left(H_{u}, T_{u}\right)+\left(H_{d}, T_{d}\right)$ & $1+1$ \\
\hline
\end{tabular}

Table 1. Spectrum for the supersymmetric SU(5) model.

instanton inducing the perturbatively missing coupling $\mathbf{1 0 1 0 5}^{\mathbf{H}}$. In contrast to GeorgiGlashow D-brane realizations, flipped SU(5) D-brane realizations, even with multiple U(1) stacks have severe phenomenological problems. Specifically, the intriguing flipped SU(5) breaking mechanism of the GUT gauge symmetry down to the standard model gauge symmetry cannot lead to a consistent low energy theory without requiring the presence of additional geometric symmetries.

The paper is organized as follows. In section 2 we analyze the Georgi-Glashow realization via 2 and 3 D-brane stacks in a bottom-up fashion. At the end we present a global three stack realization based on RCFT that exhibits a rigid $O(1)$ instanton that induces the desired coupling $\mathbf{1 0 1 0 5}^{\mathrm{H}}$. In section 3 we perform an analogous analysis for the flipped $\mathrm{SU}(5)$ model. We conclude with some final remarks in section 4. In the appendices A and B we lay out the basic ingredients for our systematic bottom-up analysis and present the results for the three-stack Georgi-Glashow realization. In appendix $\mathrm{C}$ we present all details of the global realizations including the spectrum of the hidden sector.

\section{Georgi-Glashow model}

Before turning to D-brane realizations of the Georgi-Glashow model let us briefly introduce the usual supersymmetric SU(5)-GUT model. Later on we discuss potential D-brane realizations of it. The embedding of the standard model fields is displayed in table 1.

The superpotential is given by

$$
W=10 \overline{5} \overline{5}^{\mathrm{H}}+10105^{\mathrm{H}}+\overline{\mathbf{5}} 5^{\mathrm{H}} \mathbf{1}+\mathbf{5}^{\mathrm{H}} \overline{5}^{\mathrm{H}}
$$

where the Yukawa coupling $\mathbf{1 0} \overline{\mathbf{5}} \overline{\mathbf{5}}^{\mathbf{H}}$ induces the down-flavour quark masses, the coupling $10105^{\mathrm{H}}$ the up-flavour quark and charged lepton masses, respectively, and the coupling $\overline{5} 5^{\mathrm{H}} \mathbf{1}$ the Dirac neutrino masses.

The breaking down to the standard model gauge groups occurs via an adjoint $\mathbf{2 4}$, which acquires a vev, of the form

$$
\langle\mathbf{2 4}\rangle=\operatorname{diag}\left(v, v, v,-\frac{3}{2} v,-\frac{3}{2} v\right)
$$




\begin{tabular}{|c|c|c|c|}
\hline Sector & Matter All & Transformation & Multiplicity \\
\hline$a a^{\prime}$ & $\mathbf{1 0}$ & $\boxminus_{a}$ & 3 \\
\hline$a b$ & $\overline{\mathbf{5}}$ & $(\bar{a}, b)$ & 3 \\
\hline$a b^{\prime}$ & $\mathbf{5}^{\mathbf{H}}+\overline{\mathbf{5}}^{\mathbf{H}}$ & $(a, b)+(\bar{a}, \bar{b})$ & $1+1$ \\
\hline$b b^{\prime}$ & $\mathbf{1}$ & $\bar{\square}_{b}$ & 3 \\
\hline
\end{tabular}

Table 2. Chiral spectrum for a D-brane realization of the SU(5) model.

where $v$ is of the order $10^{16} \mathrm{GeV}$. The hypercharge $\mathrm{U}(1)_{Y}$ is embedded in the $\mathrm{SU}(5)$ and given by

$$
\mathrm{U}(1)_{Y}=\operatorname{diag}\left(-\frac{1}{3},-\frac{1}{3},-\frac{1}{3}, \frac{1}{2}, \frac{1}{2}\right)
$$

which remains unbroken once the adjoint $\mathbf{2 4}$ acquires a vev of the type (2.2). After this brief introduction of the Georgi-Glashow model we turn to the D-brane realization of it.

\subsection{D-brane realization}

The most economical way to embed the Georgi-Glashow SU(5) GUT in a D-brane configuration is via two stacks of D-branes $a, b$. Stack $a$ contains 5 D-branes while stack $b$ is just a single D-brane, giving rise to the gauge symmetry $\mathrm{U}(5)_{a} \times \mathrm{U}(1)_{b}$. The abelian $\mathrm{U}(1)_{a}$ and $\mathrm{U}(1)_{b}$ are generically anomalous and become massive via the Green-Schwarz mechanism. Thus the resulting gauge symmetry is the desired $\mathrm{SU}(5)$. The massive $\mathrm{U}(1)_{a}$ and $\mathrm{U}(1)_{b}$ survive as global symmetries in the low energy effective theory and have to be preserved by all perturbative couplings.

In table 2 we display the origin of the respective matter fields for the realization of the Georgi-Glashow SU(5) model based on two stacks of D-branes. This chiral spectrum satisfies the string consistency conditions laid out in appendix A. Note that the hypercharge $\mathrm{U}(1)_{Y}$ is a subgroup of $\mathrm{U}(5)$ and thus is guaranteed to remain massless. Therefore tadpole cancellation is the only constraint one has to ensure. However, for chiral matter with the transformation property displayed in table 2 there is potentially a massless combination, satisfying the constraints (A.6) and (A.7), given by

$$
\mathrm{U}(1)_{X}=\frac{1}{4} \mathrm{U}(1)_{a}-\frac{5}{4} \mathrm{U}(1)_{b} .
$$

Let us stress that the conditions on the transformation properties of the chiral matter fields arising from tadpole cancellation and masslessness of a $\mathrm{U}(1)$ derived in the appendix A are just necessary constraints. Whether tadpoles are really cancelled and whether an abelian symmetry remains massless or not, depends crucially on the concrete global realization.

The perturbative realized Yukawa couplings are

$$
\mathbf{1 0}_{(2,0)} \overline{\mathbf{5}}_{(-1,1)} \overline{\mathbf{5}}_{(-1,-1)}^{\mathbf{H}} \quad \overline{\mathbf{5}}_{(-1,1)} \mathbf{1}_{(0,-2)} \mathbf{5}_{(1,1)}^{\mathbf{H}} \quad \mathbf{5}_{(1,1)}^{\mathbf{H}} \overline{\mathbf{5}}_{(-1,-1)}^{\mathbf{H}},
$$


where the indices indicate the charge under the global $\mathrm{U}(1)_{a}$ and $\mathrm{U}(1)_{b}$ symmetries, respectively. The Yukawa coupling

$$
\mathbf{1 0}_{(2,0)} \mathbf{1 0 _ { ( 2 , 0 ) }} \mathbf{5}_{(1,1)}^{\mathbf{H}}
$$

which contains the up-flavour quark coupling is perturbatively forbidden. An instanton with global $\mathrm{U}(1)$ charge $(-5,-1)$ under $\mathrm{U}(1)_{a}$ and $\mathrm{U}(1)_{b}$ can induce the missing coupling. As shown in [33] one needs three different instantons with global $\mathrm{U}(1)$ charge $(-5,-1)$ to give masses to all three families. Note though that the non-perturbative generation of the Yukawa coupling $\mathbf{1 0} \mathbf{1 0} \mathbf{5}^{\mathrm{H}}$ suggests that the bottom quark is heavier than the top quark, which is in contrast to experimental observations.

The perturbative realization of the Dirac neutrino mass term suggests that the neutrino masses are of the same order as the other matter field masses. However, experiments show that the neutrinos masses are $10^{-10}$ to $10^{-16}$ times smaller than the other matter field masses. The see-saw mechanism gives a natural explanation for the smallness of the neutrino masses. A necessary ingredient for the seesaw mechanism is a large Majorana mass term

$$
\mathbf{1}_{(0,-2)} \mathbf{1}_{(0,-2)}
$$

which can be induced non-perturbatively by an instanton $[15,16,23,34-37]$ with global $\mathrm{U}(1)$ charges $(0,4)$. If the Majorana mass term is in the range $\left(10^{12}-10^{15}\right) \mathrm{GeV}$ one obtains neutrino masses of the observed order $\left(10^{-2}-1\right) \mathrm{eV}$.

Let us comment on potential phenomenological drawbacks of this 2-stack quiver.

(1) The perturbatively realized coupling $\mathbf{1 0} \overline{\mathbf{5}} \overline{\mathbf{5}}^{\mathbf{H}}$ contains the Yukawa coupling giving masses to the down-flavour quarks. On the other hand the coupling $10105^{\mathrm{H}}$, which contains the Yukawa coupling that gives masses to the up-flavour quarks is perturbatively forbidden. It is induced by an instanton and thus suppressed compared to the coupling $10 \overline{\mathbf{5}} \overline{\mathbf{5}}^{\mathbf{H}}$. This suggests that for this quiver the bottom quark is heavier than the top quark which is in contrast to experimental observations.

(2) The instanton inducing the perturbatively missing Yukawa coupling $10105^{\mathbf{H}}$ also generates the dangerous dimension 5 operator $101010 \overline{5}$ [1]. For the Georgi-Glashow $\mathrm{SU}(5)$ model the dimension 5 operator $101010 \overline{5}$ includes $q_{L} q_{L} q_{L} L$ and $u_{R} u_{R} d_{R} E_{R}$, which if not sufficiently suppressed lead to a disastrous proton decay rate. Since the Yukawa coupling $10105^{\mathbf{H}}$ is responsible for the up-flavour quark coupling we expect only a minor suppression from the instanton, which is not enough to saturate the bounds on the proton lifetime.

(3) The chiral spectrum displayed in table 2 allows for a massless $\mathrm{U}(1)_{X}$ given in equation (2.4). A massless $\mathrm{U}(1)_{X}$ directly contradicts observations, and furthermore its charge explicitly forbids Majorana neutrino masses, both perturbatively and nonperturbatively. This model may be viable if the $\mathrm{U}(1)_{X}$ photon acquires a sufficiently large mass, and the mechanism responsible for that might also generate neutrino 


\begin{tabular}{|c|c|c|c|}
\hline Sector & Matter All & Transformation & Multiplicity \\
\hline$a a^{\prime}$ & $\mathbf{1 0}$ & $日_{a}$ & 3 \\
\hline$a b$ & $\overline{\mathbf{5}}$ & $(\bar{a}, b)$ & 3 \\
\hline$a c$ & $\mathbf{5}^{\mathbf{H}}+\overline{\mathbf{5}}^{\mathbf{H}}$ & $(a, \bar{c})+(\bar{a}, c)$ & $1+1$ \\
\hline$b c$ & $\mathbf{1}$ & $(\bar{b}, c)$ & 3 \\
\hline
\end{tabular}

Table 3. Spectrum for SU(5) three stack quiver with perturbative $\mu$-term.

masses, but it would be preferable to achieve all that directly in string theory. This is possible if the $\mathrm{U}(1)_{X}$ acquires a mass from axion mixing. Note that this can happen even though the conditions (A.6) and (A.7) are satisfied, since the latter are just necessary, but not sufficient, conditions to have a massless $\mathrm{U}(1)_{X} \cdot{ }^{3}$

As we will show momentarily all these problems can be overcome if one allows for an additional $\mathrm{U}(1)$ stack $c$. The $\mathrm{U}(1)_{c}$ becomes again massive via the Green-Schwarz mechanism and survives only as a global symmetry. The second problem, namely that the instanton that induces the desired Yukawa coupling $10105^{\mathrm{H}}$ generically also generates dimension five proton decay operators, can be avoided if the matter fields $\overline{\mathbf{5}}$ carry some global charge $\mathrm{U}(1)_{b}$ while the Higgs field $\mathbf{5}^{\mathbf{H}}$ is rather charged under the global $\mathrm{U}(1)_{c}$. As we show in appendix B if we furthermore want to avoid the presence of R-parity violating couplings $\mathbf{1 0} \overline{\mathbf{5}} \overline{\mathbf{5}}$ and $\overline{\mathbf{5}} \mathbf{5}^{\mathbf{H}}$ there are only two different types of choices for the origins of the fields transforming non-trivially under the $\mathrm{SU}(5)$. Within each choice there are different realizations of the Georgi-Glashow model, depending on the transformation behaviour of the right-handed neutrinos which are singlets under the $\mathrm{SU}(5)$. In appendix B we derive all three-stack quivers that mimic the Georgi-Glashow model, pass all string consistency conditions as well as some minimal phenomenological requirements.

In the following we will discuss for each type one representative. We first discuss the configuration in which the $\mu$-term is realized perturbatively

\subsubsection{Three-stack quiver with perturbatively realized $\mu$-term}

In table 3 we display the origin of the respective matter fields for a realization of the GeorgiGlashow SU(5) model based on three stacks of D-branes. It corresponds to configuration 6 in table 9 in appendix B.

In contrast to the realization based on two stacks of D-branes here we have only two perturbatively realized couplings, namely

$$
\overline{\mathbf{5}}_{(-1,1,0)} \mathbf{1}_{(0,-1,1)} \mathbf{5}_{(1,0,-1)}^{\mathbf{H}} \quad \mathbf{5}_{(1,0,-1)}^{\mathbf{H}} \overline{\mathbf{5}}_{(-1,0,1)}^{\mathbf{H}},
$$

where the subscripts again denote the respective global $\mathrm{U}(1)$ charges. The couplings

$$
\mathbf{1 0}_{(2,0,0)} \mathbf{1 0}_{(2,0,0)} \mathbf{5}_{(1,0,-1)}^{\mathbf{H}} \quad \text { and } \quad \mathbf{1 0}_{(2,0,0)} \overline{\mathbf{5}}_{(-1,1,0)} \overline{\mathbf{5}}_{(-1,0,1)}^{\mathbf{H}}
$$

\footnotetext{
${ }^{3}$ As discussed in appendix A the condition of having a massless $U(1)$ imposes constraints on the cycles the D-branes wrap. However, these constraints imply restrictions on the transformation behaviour of the chiral matter. Nevertheless the latter are just necessary conditions not sufficient.
} 
can be induced non-perturbatively via the instantons $E_{1}$ and $E_{2}$ which have global U(1)charge $(-5,0,1)$ and $(0,-1,-1)$, respectively. To get the desired hierarchy between the up-flavour and down-flavour quark masses we expect the ratio to be ${ }^{4}$

$$
e^{-S_{\text {ins }}^{E_{1}}}: e^{-S_{\text {ins }}^{E_{2}}} \simeq 100
$$

Note that the neutrino Dirac mass term is realized perturbatively. Thus in order to obtain the observed small neutrino masses we expect the presence of a large Majorana mass term for the right-handed neutrinos, which can be induced by an instanton $E_{3}$ carrying global $\mathrm{U}(1)$ charge $(0,2,-2)$. With the string scale of the order $10^{18} \mathrm{GeV}$ and a suppression factor $e^{-S_{i n s}^{E_{3}}} \simeq 10^{-5}$ we obtain via the seesaw mechanism neutrino masses in the observed range.

Let us now discuss if this setup indeed overcomes all the issues encountered for the 2 -stack realization. First note that for the three-stack setup displayed in table 3 both couplings, the $10105^{\mathrm{H}}$ as well as $\mathbf{1 0} \overline{\mathbf{5}} \overline{5}^{\mathrm{H}}$ are perturbatively forbidden. In case the suppression factor of the instanton inducing the latter one is larger than the suppression factor of the instanton generating the coupling $10105^{\mathrm{H}}$ one gets the desired hierarchy between top and bottom quark masses. Furthermore, the instanton inducing the coupling $10105^{\mathbf{H}}$ does not carry the right global charge to induce the dangerous dimension 5 operator, which could lead to a disastrous proton decay rate. Finally, this setup does not satisfy the necessary conditions on having a massless $\mathrm{U}(1)$, thus all linear combinations of $\mathrm{U}(1)_{a}, \mathrm{U}(1)_{b}$ and $\mathrm{U}(1)_{c}$ are massive. That allows the presence of the Majorana mass term for the righthanded neutrino induced by a D-instanton, which was potentially forbidden in the 2-stack realization.

\subsubsection{Three-stack quiver with non-perturbative $\mu$-term}

Again we discuss here only one representative of all the possible solutions displayed in appendix B. The chiral spectrum of this quiver is displayed in table 4 and corresponds to configuration 9 in table 10 in appendix B.

For this quiver the only perturbatively realized Yukawa coupling is

$$
\mathbf{1 0}_{(2,0,0)} \overline{\mathbf{5}}_{(-1,1,0)} \overline{\mathbf{5}}_{(-1,-1,0)}^{\mathbf{H}}
$$

which gives masses to the down-flavour quarks. The other desired couplings

$$
\begin{array}{ll}
\mathbf{1 0}_{(2,0,0)} \mathbf{1 0}_{(2,0,0)} \mathbf{5}_{(1,0,1)}^{\mathbf{H}} & \mathbf{5}_{(1,0,1)}^{\mathbf{H}} \overline{\mathbf{5}}_{(-1,-1,0)}^{\mathbf{H}} \\
\overline{\mathbf{5}}_{(-1,1,0)} \mathbf{5}_{(1,0,1)}^{\mathbf{H}} \mathbf{1}_{(0,2,0)} & \overline{\mathbf{5}}_{(-1,1,0)} \mathbf{5}_{(1,0,1)}^{\mathbf{H}} \mathbf{1}_{(0,0,2)}
\end{array}
$$

are induced via the D-instantons $E_{1}, E_{2}, E_{3}$ and $E_{4}$ which carry global U(1) charges

$$
E_{1}=(-5,0,-1) \quad E_{2}=(0,1,-1) \quad E_{3}=(0,-3,-1) \quad E_{4}=(0,-1,-3) .
$$

\footnotetext{
${ }^{4}$ To be precise the instanton $E_{1}$ induces only masses for one up-flavour quark family, thus one needs two additional instantons giving masses to the other two up-flavour quark families. Generically they have different suppression factor. Thus the ratio $e^{-S_{i n s}^{E_{1}}}: e^{-S_{i n s}^{E_{2}}} \simeq 100$ explains the hierarchy between the top and bottom-quark mass.
} 


\begin{tabular}{|c|c|c|c|}
\hline Sector & Matter All & Transformation & Multiplicity \\
\hline$a a^{\prime}$ & $\mathbf{1 0}$ & $\Xi_{a}$ & 3 \\
\hline$a b$ & $\overline{\mathbf{5}}$ & $(\bar{a}, b)$ & 3 \\
\hline$a b^{\prime}$ & $\overline{\mathbf{5}}^{\mathbf{H}}$ & $(\bar{a}, \bar{b})$ & 1 \\
\hline$a c^{\prime}$ & $\mathbf{5}^{\mathbf{H}}$ & $(a, c)$ & 1 \\
\hline$b b^{\prime}$ & $\mathbf{1}$ & $\square_{b}$ & 1 \\
\hline$c c^{\prime}$ & $\mathbf{1}$ & $\square_{c}$ & 2 \\
\hline
\end{tabular}

Table 4. Chiral spectrum for SU(5) model based on three stacks of D-branes with non-perturbative $\mu$-term.

The first term in (2.12), induced by the instanton $E_{1}$, gives masses to the up-flavour quarks as well as to the charged leptons. Since the top-quark is the heaviest Standard model field particle the suppression of the instanton must be very small. The suppression factor of $E_{2}$ on the other hand should be rather large to account for a $\mu$-term of the order $100 \mathrm{GeV}$. The instantons $E_{3}$ and $E_{4}$ induce the Dirac neutrino masses. Together with D-instantons that generate Majorana masses for the right-handed neutrinos they give via the see-saw mechanism the observed small neutrino masses. ${ }^{5}$

Let us again discuss whether this quiver indeed overcomes all the issues encountered for the two stack quiver. The major drawback of the two-stack quiver, namely that the instanton that induces the desired Yukawa coupling $10105^{\mathbf{H}}$ also generates the dangerous dimension five operator $101010 \overline{\mathbf{5}}$, is not a problem for this quiver. Also this quiver like the 3-stack quiver discussed before does not exhibit any abelian symmetry that remains massless. Thus all perturbatively missing terms can be generated via D-instantons. However the perturbative realization of down flavour quark masses compared to the nonperturbative up flavour quark mass suggests exactly the opposite mass hierarchy compared to the observed one. Let us point out though that both couplings are further suppressed via world-sheet instantons which after all can in principle suppress the down-flavour quark masses such that the top-quark is indeed as observed the heaviest standard model particle. The latter is however not the generic case and usually requires some amount of fine-tuning.

\subsection{Global realization of a three-stack quiver}

Here we present a global realization of a three-stack quiver which is similar to the ones we discussed above. We will see that one can indeed find a rigid $O(1)$ instanton that exhibits the correct zero mode structure to induce the perturbatively forbidden Yukawa coupling $10105^{\mathbf{H}}$. The model is based on RCFT which are called Gepner orientifolds.

Gepner orientifolds are constructed by replacing the geometric notion of curled extra dimensions to form a compact manifold, by an algebraic procedure where the internal sector consists of tensor products of $\mathcal{N}=2$ minimal superconformal models with total central

\footnotetext{
${ }^{5}$ Large suppression factors of $E_{3}$ and $E_{4}$ can account for the smallness of the neutrino mass [38]. In that case no Majorana masses for the right-handed neutrinos should be generated.
} 
charge $c=9$ [39-44]. ${ }^{6}$ In this context, there has been an extensive search for all possible embeddings of the standard model gauge theory in D-brane configurations [48-51].

Before presenting a concrete example let us describe our search for realistic three-stack quivers, which is based on a previous study performed in [50]. There the authors searched first for local configurations of D-brane boundary states that reproduce the chiral spectrum of the MSSM or extensions of it, such as SU(5) GUT's. These local D-brane boundary state configurations were required to not saturate the tadpole constraints and moreover give rise to a hypercharge embedding that is compatible with the MSSM hypercharge assignment and does not become massive via the Green-Schwarz mechanism. In a few cases the Dbrane boundary state configuration that gives rise to the MSSM, called visible sector in the following, was enough to satisfy the tadpole constraints. However, generically one needs additional boundary states to cancel the tadpoles. In the search performed in [50] the authors required that these additional boundary states, usually called hidden sector, are added in such a way that one does not have any chiral matter fields charged with respect to gauge groups in the visible and hidden sector, simultaneously. With this approach the authors found many globally consistent configurations, that give realistic chiral spectra, where the latter include also SU(5) GUT and Pati-Salam-realizations.

In this work we follow a similar path. We take the subset of local configurations that give rise to a $\mathrm{SU}(5)$ GUT-like spectrum and analyze the superpotential by looking at the global U(1) charges of the respective matter fields. In case a desired Yukawa coupling is missing we are looking for a rigid $O(1)$ instanton that has the correct zero mode structure to induce the missing coupling. To be more precise we identify all instanton boundary states that are orientifold invariant and do not exhibit any additional neutral fermionic zero modes apart from the two universal $\theta^{\alpha}$ modes [20-23]. Then we further require that the intersection pattern of this instanton boundary state with the visible D-brane boundary state configuration is in such a way that it gives the correct charged zero mode structure to induce the perturbatively forbidden, but desired coupling.

Once such an instanton is found we are looking for a hidden sector that cancels the tadpoles in such a way that it does not intersect with the instanton. This way it is ensured that the instanton does not exhibit any additional zero modes charged with respect to the hidden sector, which would kill the instanton contribution to the perturbatively missing coupling. In general, an already known solution to the tadpole cancellation condition is not very likely to satisfy this criterion, so one usually has to perform a new search for hidden sectors, imposing the instanton zero-mode constraint. Note that this constraint is rather strong, since it demands complete absence of zero-modes, even vector-like ones.

In addition to intersecting the instanton brane, the hidden sector branes may also intersect the observable matter brane. This is usually indeed what happens, although there are rare examples where there is no massless observable-hidden matter at all [50], or where no hidden sector is needed to cancel all tadpoles [49]. In the latter case, the problem is of course already solved, but then one looses the possibility of using the hidden sector for supersymmetry breaking. Apart from these rare cases, the common procedure

\footnotetext{
${ }^{6}$ For some initial studies on closed Gepner constructions see [45-47].
} 
is to allow observable-hidden matter, provided it is completely vector-like with respect to the entire gauge group. In that case, the additional matter may acquire a mass without any gauge symmetry breaking. In principle, one may relax the above restriction further and even allow observable-hidden matter that is chiral, but that becomes vector-like if the hidden sector gauge symmetry is removed. Then it depends on further details of the hidden sector dynamics what ultimately happens to the exotic observable-hidden matter, but it is not difficult to think of scenarios where it becomes sufficiently massive. In explicit examples, the number of tadpole solutions increases by several orders of magnitude under these relaxed conditions in comparison to the strict ones (i.e. those allowing only vectorlike observable-hidden matter). Our attitude here is that chiral observable-hidden matter of this kind is a lesser evil than superfluous instanton zero modes, and therefore we use the relaxed condition, after checking that the strict one does not generate any solutions. Previous searches have shown that under the strict observable-hidden conditions, instantons with the correct zero mode structure to generate desired interactions $[1,23]$ are very rare.

In [50] the authors found 7 different semi-realistic three-stack realizations of the SU(5) GUT's. In this search they allowed also for $O(1)$ gauge groups for the additional third D-brane stack. Let us also point out that this subset mainly contains setups in which the $\mu$ term is perturbatively forbidden. ${ }^{7}$ Thus configurations of the type discussed 2.1.1 are not contained in the search we will perform. We leave it for future work to extend the search of three-stack quivers by also including quivers in which the $\mu$-term is perturbatively realized.

Performing the analysis in the fashion described above we find one type of configuration that gives rise to a semi realistic model and exhibits a rigid $O(1)$ instanton generating the perturbatively forbidden Yukawa coupling $10105^{\mathbf{H}}$. The visible sector consists of three stacks of branes giving rise to the gauge symmetry

$$
\mathrm{U}(5)_{a} \times \mathrm{U}(1)_{b} \times O(1)_{c}
$$

The spectrum of a specific model is displayed in table 5 , where any constituent D-brane boundary state of the hidden sector is denoted by $h$. For the sake of clarity we do not display any specifics of the hidden sector. For the details, such as the hidden sector gauge symmetry as well as the spectrum within the hidden sector we refer to appendix C.

Note that for this particular configuration there are three pairs of Higgs and no neutrinos. Moreover the hidden sector intersects chirally with the visible sector, giving rise to exotics. The net number of $\mathrm{SU}(5)$ exotics is as expected zero, thus they are non-chiral with respect to the GUT gauge symmetry. However, the exotics carry different charge with respect to the hidden gauge groups. They only acquire mass after a breakdown of the hidden gauge group or via D-instantons inducing mass terms for them.

Let us turn to the Yukawa couplings. The only perturbatively realized coupling is the

\footnotetext{
${ }^{7}$ In the search performed in [50] three-stack quivers with a perturbatively realized $\mu$-term were considered as two stack quivers, rather than three stack quivers, due to the fact that the chiral spectrum arises from only two stacks. From the available data we can therefore not decide if a third brane with the right properties can be found.
} 


\begin{tabular}{|c|c|c|c|}
\hline Sector & Matter All & Transformation & Multiplicity \\
\hline$a a^{\prime}$ & $\mathbf{1 0}$ & $\exists_{a}$ & 3 \\
\hline$a b$ & $\overline{\mathbf{5}}$ & $(\bar{a}, b)$ & 3 \\
\hline$a b^{\prime}$ & $\overline{\mathbf{5}}^{\mathbf{H}}$ & $(\bar{a}, \bar{b})$ & 3 \\
\hline$a c$ & $\mathbf{5}^{\mathbf{H}}$ & $(a, c)$ & 3 \\
\hline$a h$ & $\mathbf{5}^{\mathrm{ex}}$ & $(a, h)$ & 4 \\
\hline$a h$ & $\overline{\mathbf{5}}^{\mathrm{ex}}$ & $(\bar{a}, h)$ & 4 \\
\hline
\end{tabular}

Table 5. Visible spectrum of a globally consistent SU(5) model based on three stacks of D-branes.

down flavour coupling

$$
\mathbf{1 0}_{(2,0,0)} \overline{\mathbf{5}}_{(-1,1,0)} \overline{\mathbf{5}}_{(-1,-1,0)}^{\mathbf{H}} .
$$

For the up flavour Yukawa coupling

$$
\mathbf{1 0}_{(2,0,0)} \mathbf{1 0}_{(2,0,0)} \mathbf{5}_{(1,0,1)}^{\mathbf{H}}
$$

which is perturbatively absent we find an instanton that wraps a rigid orientifold invariant cycle that has the following intersection pattern with the visible branes

$$
\pi_{E} \circ \pi_{a}=1 \quad \pi_{E} \circ \pi_{b}=0 \quad \pi_{E} \circ \pi_{c}=1
$$

Thus it gives the correct uncharged and charged zero mode structure to induce the desired but perturbatively missing coupling. Note also that this instanton does not intersect with any of the hidden D-brane boundary states and thus does not exhibit any zero modes charged with respect to the hidden sector that would spoil the generation of $10105^{\mathbf{H}}$. The suppression of the instanton turns out to be too large to account for the observed masses of the standard model. Let us stress though that this analysis is performed at the exact RCFT point and moving away from this exact point in moduli space might improve the situation.

Let us turn to the $\mu$-term

$$
\mathbf{5}_{(1,0,1)}^{\mathbf{H}} \overline{\mathbf{5}}_{(-1,-1,0)}^{\mathbf{H}}
$$

which is perturbatively forbidden and can be generated by an instanton with the intersection pattern

$$
\pi_{E} \circ \pi_{a}=0 \quad \pi_{E} \circ \pi_{b}=-1 \quad \pi_{E} \circ \pi_{c}=1
$$

In order to be compatible with phenomenology one expects the instanton to exhibit a large suppression factor. Unfortunately, for this specific example we do not find any rigid $O(1)$ instantons with such intersection pattern. 


\begin{tabular}{|c|c|c|c|}
\hline Representation & SM matter embedding & Multiplicity & $\mathrm{U}(1)_{X}$ \\
\hline $\mathbf{1 0}$ & $\left(q_{L}, d_{R}, \nu_{R}\right)$ & 3 & $\frac{1}{2}$ \\
\hline$\overline{\mathbf{5}}$ & $\left(L, u_{R}\right)$ & 3 & $-\frac{3}{2}$ \\
\hline $\mathbf{1}$ & $e_{R}$ & 3 & $\frac{5}{2}$ \\
\hline $\mathbf{5}_{\mathbf{H}}+\overline{\mathbf{5}}_{\mathbf{H}}$ & $\left(H_{d}, T_{d}\right)+\left(H_{u}, T_{u}\right)$ & $1+1$ & -1 \\
\hline $\mathbf{1 0}_{\mathbf{H}}+\overline{\mathbf{1 0}}_{\mathbf{H}}$ & $(\Delta)+(\bar{\Delta})$ & $1+1$ & $\frac{1}{2}-\frac{1}{2}$ \\
\hline
\end{tabular}

Table 6. Spectrum for the D-brane realization of the flipped SU(5) model.

Despite its phenomenological problems, such as absence of $\mu$-terms and Dirac mass term for the neutrinos, a highly suppressed top-quark mass, as well as the presence of additional exotics that are chiral with respect to the hidden sector but not with respect to the $\mathrm{SU}(5)$, this configuration serves as global realization of the $\mathrm{SU}(5)$ quivers discussed above in which one can find an instanton that satisfies the severe zero mode constraints to induce a perturbatively missing coupling $10105^{\mathbf{H}}$. Let us stress again that we looked only in a small phenomenologically interesting subset of $\mathrm{SU}(5)$ quivers while other appealing quivers with a perturbatively realized $\mu$-term were not covered by this search. Additionally, we only allowed for rigid $O(1)$ instantons to give non-perturbative contributions to the superpotential. However, as shown in [24-27] also multi-instanton configurations and so called rigid U(1) instantons [25, 28-32] can generate some of the missing Yukawa couplings. We leave it for future work to extend the here performed analysis by extending the class of local configurations and by including additional effects for the non-perturbative generation of desired couplings.

\section{$3 \quad$ Flipped SU(5) model}

In this section we discuss the realization of supersymmetric flipped SU(5)-GUT models in orientifold models. Before we present and analyze specific D-brane configurations which give rise to flipped $\mathrm{SU}(5)$ gauge theory in four-dimensional space-time let us give a brief introduction to the flipped SU(5) model. It consists of a non-abelian part SU(5) accompanied with an abelian $\mathrm{U}(1)_{X}$ gauge symmetry. The standard model matter fields appear as antisymmetric $\mathbf{1 0}_{\frac{1}{2}}$, anti-fundamental $\overline{\mathbf{5}}_{-\frac{3}{2}}$ and singlet $\mathbf{1}_{\frac{5}{2}}$ under the $\mathrm{SU}(5)$, where the subscript denote the charge of the respective representation under the $\mathrm{U}(1)_{X}$. In table 6 we present the embedding of the standard model fields into the flipped SU(5) multiplets. In addition to the electroweak Higgs fields $\boldsymbol{5}_{\mathbf{H}}$ and $\overline{\mathbf{5}}_{\mathbf{H}}$ the flipped SU(5) model also contains the Higgs fields $\mathbf{1 0}_{\mathbf{H}}$ and $\overline{\mathbf{1 0}}_{\mathbf{H}}$, whose presence is crucial for the breaking mechanism of the GUT gauge symmetry down to the Standard model gauge symmetry. Note that the spectrum assignment is similar to the one of the Georgi-Glashow model with the exchange

$$
u_{R} \leftrightarrow d_{R} \quad e_{R} \leftrightarrow \nu_{r} \quad H_{u} \leftrightarrow H_{d}
$$




\begin{tabular}{|c|c|c|c|c|}
\hline Sector & Matter All & Transformation & Multiplicity & $\mathrm{U}(1)_{X}$ \\
\hline$a a^{\prime}$ & 10 & $日_{a}$ & 3 & $\frac{1}{2}$ \\
\hline$a b$ & $\overline{5}$ & $(\bar{a}, b)$ & 3 & $-\frac{3}{2}$ \\
\hline$a b^{\prime}$ & $5^{\mathrm{H}}+\overline{5}^{\mathrm{H}}$ & $(a, b)+(\bar{a}, \bar{b})$ & $1+1$ & $\begin{array}{ll}-1 & 1\end{array}$ \\
\hline$b b^{\prime}$ & 1 & $\bar{\square}_{b}$ & 3 & $\frac{5}{2}$ \\
\hline$a a^{\prime}$ & $10^{\mathrm{H}}+\overline{\mathbf{1 0}}^{\mathrm{H}}$ & $\theta_{a}+\bar{\theta}_{a}$ & $1+1$ & $\frac{1}{2}-\frac{1}{2}$ \\
\hline
\end{tabular}

Table 7. Chiral spectrum for the flipped SU(5) model.

The hypercharge is a subgroup of $\mathrm{SU}(5) \times \mathrm{U}(1)_{X}$, given by

$$
\mathrm{U}(1)_{Y}=-\frac{1}{5} \operatorname{diag}\left(-\frac{1}{3},-\frac{1}{3},-\frac{1}{3}, \frac{1}{2}, \frac{1}{2}\right)+\frac{2}{5} \mathrm{U}(1)_{X} .
$$

In addition to the gauge symmetries there is a discrete $\mathbf{Z}_{2}$ symmetry $\mathbf{1 0}^{\mathbf{H}} \rightarrow-\mathbf{1 0}^{\mathrm{H}}$. Then the superpotential takes the form

$$
W=10 \overline{5} \overline{5}^{\mathrm{H}}+10105^{\mathrm{H}}+\overline{\mathbf{5}} 5^{\mathrm{H}} \mathbf{1}+\mathbf{5}^{\mathrm{H}} \overline{\mathbf{5}}^{\mathrm{H}}+10^{\mathrm{H}} 10^{\mathrm{H}} 5^{\mathrm{H}}+\overline{\mathbf{1 0}}^{\mathrm{H}} \overline{\mathbf{1 0}}^{\mathrm{H}} \overline{5}^{\mathrm{H}} .
$$

Here the first three terms give masses to the standard fields after the electroweak Higgses acquire a vev, the fourth term is the $\mu$-term, and the last two terms are crucial for the doublet-triplet splitting after the component $\Delta_{45}$ and $\bar{\Delta}_{45}$ of $\mathbf{1 0}^{\mathbf{H}}$ and $\overline{\mathbf{1 0}}^{\mathbf{H}}$, respectively, acquire a vev of the GUT scale.

\subsection{D-brane realization}

Again the most economical way to embed the flipped SU(5) model in a D-brane configuration is via two stacks of D-branes $a, b$. Stack $a$ contains 5 D-branes while stack $b$ consists of just a single D-brane. Thus the resulting gauge symmetry is then $\mathrm{U}(5)_{a} \times \mathrm{U}(1)_{b}$, where the abelian $\mathrm{U}(1)_{a}$ and $\mathrm{U}(1)_{b}$ are generically anomalous and become massive via the Green-Schwarz mechanism. However, in order to mimic the flipped SU(5) model the linear combination

$$
\mathrm{U}(1)_{X}=\frac{1}{4} \mathrm{U}(1)_{a}-\frac{5}{4} \mathrm{U}(1)_{b}
$$

has to remain massless, thus has to satisfy the constraints (A.6) and (A.7) displayed in appendix A. In table 7 we display the origin of the respective matter fields for the realization of the flipped SU(5) model based on two stacks of D-branes.

Let us again discuss the superpotential terms, beginning with the terms that give eventually masses to the standard model fields. The perturbatively realized couplings are

$$
\mathbf{1 0}_{(2,0)} \overline{\boldsymbol{5}}_{(-1,1)} \overline{\boldsymbol{5}}_{(-1,-1)}^{\mathbf{H}} \quad \overline{\mathbf{5}}_{(-1,1)} \mathbf{1}_{(0,-2)} \mathbf{5}_{(1,1)}^{\mathbf{H}} \quad \mathbf{5}_{(1,1)}^{\mathbf{H}} \overline{\boldsymbol{5}}_{(-1,-1)}^{\mathbf{H}} .
$$

They contain the Yukawa couplings that give masses to the up-flavour quarks, the charged leptons as well as the neutrinos and also the $\mu$-term. Here the subscripts denote again the 
charges under the global U(1)'s. However the coupling

$$
\mathbf{1 0} \mathbf{0}_{(2,0)} \mathbf{1 0}(2,0) \mathbf{5}_{(1,1)}^{\mathbf{H}}
$$

whose presence is required to give masses to the down-flavour quarks is perturbatively forbidden. It can be generated by an instanton which carries the charge $(-5,-1)$ under the global U(1)'s. Since the instanton induced Yukawa matrix factorizes one needs three different instantons to generate masses for all three families. Thus the non-perturbative nature of the coupling $10105^{\mathrm{H}}$ cannot only explain the observed mass hierarchy between top and bottom quarks but also potentially explains the hierarchy between the different down-flavour families.

Let us now turn to the superpotential terms which are crucial for the GUT-breaking down to the Standard model gauge symmetry. Both terms

$$
\mathbf{1 0}_{(2,0)}^{\mathbf{H}} \mathbf{1 0}_{(2,0)}^{\mathbf{H}} \mathbf{5}_{(1,1)}^{\mathbf{H}} \quad \overline{\mathbf{1 0}}_{(-2,0)}^{\mathbf{H}} \overline{\mathbf{1 0}}_{(-2,0)}^{\mathbf{H}} \overline{\mathbf{5}}_{(-1,-1)}^{\mathbf{H}}
$$

whose presence is crucial are perturbatively forbidden. While the first one will be generated by the same instanton which also generates the Yukawa coupling $10105^{\mathbf{H} 8}$ an instanton with charge $(5,1)$ under the global $\mathrm{U}(1)$ charges can induce the perturbatively missing coupling $\overline{\mathbf{1 0}} \mathbf{H} \overline{\mathbf{1 0}} \mathbf{H} \overline{\mathbf{5}}^{\mathbf{H}}$.

While this D-brane quiver after taking into account the non-perturbative effects can in principle mimic the flipped SU(5) model it has some phenomenological flaws, which we will discuss below.

(1) The perturbatively realized Yukawa coupling $\mathbf{1 0} \overline{\mathbf{5}} \overline{\mathbf{5}}^{\mathbf{H}}$ contains the Yukawa couplings giving masses to the up-flavour quarks and the neutrinos. Thus they are expected to be of the same order which is in contradiction to experiments that observe a hierarchy of $10^{-16}$ between the top- quark mass and the neutrino masses. Note though that this is not a problem due to the D-brane realization but rather a problem within the flipped SU(5) model. In [52] the authors present a flipped SU(5) model which allows for additional singlets $\Phi$, which are uncharged under the $\mathrm{SU}(5)$ as well as under the $\mathrm{U}(1)_{X}$. These singlets couple to the left-handed neutrinos via the coupling $\overline{\mathbf{1 0}}^{\mathbf{H}} \mathbf{1 0} \Phi$. After the $\overline{\mathbf{1 0}}^{\mathbf{H}}$ gets a vev on the order of the GUT-scale the Yukawa coupling effectively becomes a large Majorana neutrino mass which via the seesaw mechanism may explain the smallness of the neutrino masses. However for the Dbrane realization of the flipped SU(5) model with only two D-brane stacks one cannot accommodate a matter field which is not charged under the $\mathrm{SU}(5)$ and the $\mathrm{U}(1)_{X} \cdot{ }^{9}$

\footnotetext{
${ }^{8}$ This coupling will be actually induced by a fourth instanton with the same charge under the global

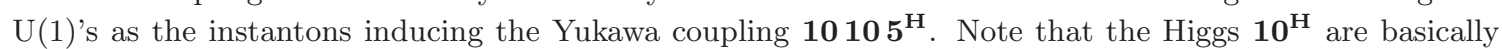
a fourth family and thus in order to induce the Yukawa coupling $10105^{\mathbf{H}}$ for all three families as well as for the Higgs fields $\mathbf{1 0}^{\mathbf{H}}$ one needs four different instantons with the global U(1)-charge $(-5,-1)$. Note also that this potentially implies that one has to perform a field redefinition in order to have the correct superpotential.

${ }^{9}$ In principle the desired singlet could be an open string moduli transforming as an adjoint under the $\mathrm{U}(1)_{b}$.
} 
(2) As already discussed in [1] an instanton inducing the coupling $10105^{\mathbf{H}}$ also generates the dimension 5 operator $101010 \overline{\mathbf{5}}$. The latter contains the dangerous dimension 5 operator $q_{L} q_{L} q_{L} L$ which if not sufficiently suppressed leads to a disastrous proton decay rate. To match the observed hierarchy between the top-quark and bottomquark mass we expect the instanton suppression on the order of $10^{-2}$, which is not enough to saturate the bounds on the proton lifetime. Moreover, in the quiver displayed in table 7 the dimension 5 operator $10 \overline{5} \overline{5} 1$ is perturbatively realized. This operator includes the dimension five operator $u_{R} u_{R} d_{R} E_{R}$, which also has to be highly suppressed to saturate the bounds on the proton lifetime. Since it is perturbatively realized and thus only suppressed by the string scale $M_{s}$ it poses a serious phenomenological problem and predicts a proton lifetime not compatible with experimental observations.

(3) The quiver displayed in table 7 generically predicts the presence of the terms

$$
\mathbf{1 0}_{(2,0)} \overline{\mathbf{1 0}}_{(-2,0)}^{\mathbf{H}} \quad \mathbf{1 0}_{(2,0)}^{\mathbf{H}} \overline{\mathbf{1 0}}_{(-2,0)}^{\mathbf{H}} .
$$

Note that only one linear combination $\widetilde{\mathbf{1 0}}=\sum_{I} c_{I} \mathbf{1 0}^{I}+c_{H} \mathbf{1 0 ^ { \mathrm { H } }}$, where $I$ runs over all three families, becomes massive. However, independent on whether the linear combination $\widetilde{\mathbf{1 0}}$ is interpreted as the Higgs $\mathbf{1 0}^{\mathbf{H}}$ or as one of the three family matter fields $\mathbf{1 0}$ the presence of a mass term of the form (3.8) poses serious problems. In the latter case it would induce a tadpole after $10^{\mathrm{H}}$ acquires a vev, indicating an instability of the vacuum. For the former situation in which $\widetilde{\mathbf{1 0}}$ is interpreted as the Higgs $1 \mathbf{1 0}^{\mathbf{H}}$ the mass term would forbid the simultaneous acquirement of a vev for $\mathbf{1 0} \mathbf{H}$ and $\overline{\mathbf{1 0}}^{\mathbf{H}}$, otherwise supersymmetry is broken at the GUT scale.

Note, however that the mass term $\mathbf{1 0}^{\mathbf{H}} \overline{\mathbf{1 0}}^{\mathbf{H}}$ is induced via the three-point couplings

$$
\left(\Phi_{5}-\Phi_{1}\right) \overline{\mathbf{1 0}}^{\mathbf{H}} \mathbf{1 0}^{\mathbf{H}}
$$

where $\Phi_{5}$ and $\Phi_{1}$ denote the scalar fields transforming in the adjoint of the overall $\mathrm{U}(1)$ of the $\mathrm{U}(5)$ D-brane stack and of the U(1) D-brane stack. These vevs are related to the position in the internal space and in case they take the same value the mass is zero and the problematic term (3.8) is absent. Generically it requires a large amount of fine-tuning to avoid the presence of the mass terms of the form (3.8).

(4) In the quiver displayed in table 7 the coupling

$$
\mathbf{1 0}_{(2,0)}^{\mathbf{H}} \overline{\mathbf{5}}_{(-1,1)} \overline{\mathbf{5}}_{(-1,-1)}^{\mathbf{H}}
$$

is perturbatively realized. Note that the field redefinition which is necessary to ensure that only the Yukawa couplings $10105^{\mathrm{H}}$ and $10^{\mathrm{H}} \mathbf{1 0}^{\mathrm{H}} 5^{\mathrm{H}}$ are present but no mixed terms $1 \mathbf{1 0}^{\mathrm{H}} \mathbf{1 0} \mathbf{5}^{\mathrm{H}}$, which would lead to large masses for some of the MSSM matter fields cannot ensure the absence of the Yukawa coupling (3.10) (see footnote 8). However, after the component $\Delta_{45}$ of $\mathbf{1 0}^{\mathbf{H}}$ acquires a vev of the GUT scale the presence of the term (3.10) would give rise to a large R-parity violating term $H_{u} L$, which is not compatible with experimental observations. 
While the problem (3) can be avoided with some amount of fine-tuning the issues (1) and (2) can be overcome by allowing for another U(1) brane stack, analogously to the Georgi-Glashow D-brane realization. However, even in a three-stack realization one faces the serious issue of the presence of the superpotential term $10^{\mathbf{H}} \overline{\mathbf{5}} \overline{\mathbf{5}}^{\mathrm{H}}$ that leads to the large R-parity violating term $L H_{u}$, thus giving $H_{u}$ and $L$ mass of the order of $M_{G U T}$, after the component $\Delta_{45}$ of $\mathbf{1 0} \mathbf{H}$ acquires a vev of the GUT scale.

For specific string compactifications there may exist additional symmetries which emerge from the compactification manifold. In case such a symmetry forbids the undesired couplings the quiver displayed in table 7 is a viable D-brane configuration. However, let us emphasize that such symmetries may also forbid some of the desired couplings. Moreover, for a generic compactification we do not expect such symmetries to appear.

Summarizing we have shown that D-brane realization of the flipped SU(5) has serious phenomenological problems. Some of the problems can be overcome by allowing additional D-brane stacks. However for a generic string embedding the D-brane quivers mimicking the flipped $\mathrm{SU}(5)$ model exhibit the superpotential term $10^{\mathbf{H}} \overline{\mathbf{5}} \overline{\mathbf{5}}^{\mathbf{H}}$, that gives rise to an R-parity violating term of the order of $M_{G U T}$.

\section{Conclusions}

In this work we discussed the realization of SU(5) GUT's in the framework of orientifold compactifications. We analyze how in such compactifications the superpotential can be accommodated, where we assume that perturbatively non-realized couplings are generated via D-instanton effects. Often times the D-instanton that induces a desired Yukawa coupling also generates a coupling that poses phenomenological problems. For the SU(5) orientifold realizations the coupling $10105^{\mathbf{H}}$ is perturbatively forbidden, and thus needs to be realized non-perturbatively. However, in the most economical SU(5) realization the D-instanton giving rise to the $\mathbf{1 0 1 0 5}^{\mathbf{H}}$ induces also the dangerous dimension 5 operator $101010 \overline{5}$. The presence of the latter would lead to a disastrous proton decay rate.

We show that this problem can be overcome by allowing for an additional D-brane stack. We display viable SU(5) quivers based on three stacks of D-branes and investigate them with respect to their phenomenology. Furthermore, we present global Gepner model realizations of these quivers. These models exhibit D-instantons that satisfy the severe constraints on the zero mode structure to induce the coupling $\mathbf{1 0 1 0 5}^{\mathbf{H}}$. Unfortunately the instanton suppressions are too high to be phenomenologically viable. Nevertheless, these examples serve as global realization of the phenomenological viable $\mathrm{SU}(5)$ quivers. The performed search of global realizations contained only a small subset of viable quivers and it would be interesting to extend the search by also allowing quivers with a perturbatively realized $\mu$-term.

Finally, we perform an analogous analysis for flipped SU(5) models. In the absence of any additional geometric symmetries of the compactification manifold D-brane quivers mimicking the flipped SU(5) model exhibit severe phenomenological problems. The $\mathbf{1 0}^{\mathbf{H}}$ required for the intriguing $\mathrm{SU}(5)$ breaking mechanism generically couples to the standard 
model fields. After acquiring a vev of the GUT scale it induces large masses for the standard model fields not compatible with observations.

\section{Acknowledgments}

We would like to acknowledge interesting discussions and correspondence with M. Bianchi, M. Cvetič, J. Halverson, A. Lionetto, J.F. Morales, E. Kiritsis and N. D. Vlachos. P.A. would like to thank University of Crete, LP Ecole Normale Supérieure de Lyon, Ecole Polytechnique and CERN for hospitality during the last stage of this work. R.R. and A.N.S. are grateful to KITP Santa Barbara for hospitality during parts of this work. This research is supported by the Dutch Foundation for Fundamental Research of Matter (FOM) as part of the program STQG (String Theory and Quantum Gravity, FP 57). This work has been partially supported by funding of the European Research and Training Network (RTN) grant "Unification in the LHC era "(PITN-GA-2009-237920), the Spanish Ministerio de Ciencia e Innovación, Research Project FPA2008-02968, and by the Project CONSOLIDER-INGENIO 2010, Programme CPAN (CSD2007-00042).

\section{A String consistency conditions and phenomenological constraints}

In this appendix, we briefly summarize string consistency conditions that D-brane quivers have to satisfy. The latter contain constraints arising from tadpole cancellation and constraints that have to be fulfilled in case a linear combination $\sum_{x} q_{x} \mathrm{U}(1)_{x}$ should remain massless and thus survive as abelian gauge symmetry in the low energy effective action. For a more detailed description, we refer the reader to [53] (for an analogous analysis see $[49,54]) \cdot{ }^{10}$

\section{A.1 Tadpole cancellation}

The tadpole cancellation condition, given by

$$
\sum_{x} N_{x}\left(\pi_{x}+\pi_{x}^{\prime}\right)=4 \pi_{O}
$$

is a condition on the cycles that the D-branes wrap. Here $\pi_{x}, \pi_{x}^{\prime}$ and $\pi_{O}$ denote the homology class of the cycles the brane $x$, its orientifold image $x^{\prime}$ and the orientifold $O$ wrap. Moreover, $N_{x}$ is the number of D-brane for stack $x$. Multiplying the tadpole cancellation condition with the homology class $\pi_{a}$ corresponding to the cycle wrapped by the D-brane stack $a$ and using the chiral spectrum displayed in table 8 one derives constraints on the transformation behaviour of the chiral matter given by

$$
\#\left(\square_{a}\right)+\left(N_{a}-4\right) \#\left(\square_{a}\right)+\left(N_{a}+4\right) \#\left(\square_{a}\right)=0,
$$

Note that for $N_{a}>2$ this condition is the usual anomaly cancellation condition for nonabelian $\mathrm{SU}\left(N_{a}\right)$ gauge symmetries. For $N_{a}=2$ it is a string-theoretic condition for antisymmetric $\mathrm{U}(2)$ tensors that does not correspond to any field-theoretic anomaly condition.

\footnotetext{
${ }^{10}$ For analogous work see $[1,32,37,55-61]$. First local (bottom-up) constructions were discussed in [62$64]$.
} 


\begin{tabular}{|c|c|}
\hline Representation & Multiplicity \\
\hline$\square_{a}$ & $\frac{1}{2}\left(\pi_{a} \circ \pi_{a}^{\prime}+\pi_{a} \circ \pi_{\mathrm{O} 6}\right)$ \\
$\square_{a}$ & $\frac{1}{2}\left(\pi_{a} \circ \pi_{a}^{\prime}-\pi_{a} \circ \pi_{\mathrm{O} 6}\right)$ \\
$\left(\square_{a}, \bar{\square}_{b}\right)$ & $\pi_{a} \circ \pi_{b}$ \\
$\left(\square_{a}, \square_{b}\right)$ & $\pi_{a} \circ \pi_{b}^{\prime}$ \\
\hline
\end{tabular}

Table 8. Chiral spectrum.

However, since these anti-symmetric tensors carry a charge under the phase symmetry of $\mathrm{U}(2)$, they can be distinguished from $\mathrm{SU}(2)$ singlets. Therefore this condition can be imposed on the field theory spectrum, and it must be imposed to have any chance to find a string theory embedding. For $N_{a}=1$ the anti-symmetric tensor cannot even be detected in the massless spectrum, and hence a given field theory spectrum may correspond to a string theory spectrum with any number of chiral anti-symmetric tensors (where "chiral" is defined as for $N_{a}>2$ ), which are infinite towers with a vanishing ground state dimension. However, since that number must be an integer, this still imposes a condition

$$
\#\left(\square_{a}\right)+5 \#\left(\square_{a}\right)=0 \quad \bmod 3 .
$$

\section{A.2 Massless U(1)'s}

In order to have a massless linear combination $\mathrm{U}(1)^{11}$

$$
\mathrm{U}(1)=\sum_{x} q_{x} \mathrm{U}(1)_{x}
$$

the cycles that the D-brane stacks $x$ wrap have to satisfy [63]

$$
\sum_{x} q_{x} N_{x}\left(\pi_{x}-\pi_{x}^{\prime}\right)=0 .
$$

Analogously to the tadpole cancellation, multiplying both sides with the homology class $\pi_{a}$ and using the relations displayed in table 8 one obtains constraints on the transformation properties of the chiral matter. They take the form

$$
\sum_{x \neq a} q_{x} N_{x} \#\left(\square_{a}, \square_{x}\right)-\sum_{x \neq a} q_{x} N_{x} \#\left(\square_{a}, \square_{x}\right)=q_{a} N_{a}\left(\#\left(\square_{a}\right)+\#\left(\square_{a}\right)\right)
$$

for $N_{a}>1$. The case $N_{a}=1$ requires a little more care due to the fact that in massless spectrum the antisymmetric tensor is absent. Using (A.2) to express the "would be" antisymmetrics in terms of the fundamentals and symmetrics one obtains

$$
\sum_{x \neq a} q_{x} N_{x} \#\left(\square_{a}, \bar{\square}_{x}\right)-\sum_{x \neq a} q_{x} N_{x} \#\left(\square_{a}, \square_{x}\right)=q_{a} \frac{\#\left(\square_{a}\right)+8 \#\left(\square_{a}\right)}{3} .
$$

\footnotetext{
${ }^{11}$ Note that higher-dimensional anomalies might affect the four-dimensional theory upon decompactifications and render masses to gauge bosons which are free of four dimensional anomalies [65-68].
} 
Since the flipped SU(5) model requires an additional abelian gauge symmetry, namely $\mathrm{U}(1)_{X}$ we require these constraints on the chiral spectrum of the flipped $\mathrm{SU}(5)$ model to be satisfied by the linear combination $\mathrm{U}(1)_{X}$.

\section{A.3 Derivation for RCFT models}

The foregoing derivations were made using the language of D-branes wrapping cycles on a manifold. Here we will show how the same formulas can be derived using boundary and crosscap states on RCFT orientifolds. For equation (A.2) it suffices to refer to [54]. In [48] this was worked out for the simple current boundary state formalism developed in [69].

Equation (A.6) can be derived as follows. The condition that a massless U(1) boson does not couple to an RR-axion is [48]

$$
\sum_{x} q_{x} N_{x}\left(R_{x(m, J)}-R_{x^{c}(m, J)}\right)=0
$$

where $q_{x}$ and $N_{x}$ are as above, and $R_{x(m, J)}$ are the boundary coefficients as defined in [69]. Here $x$ labels distinct boundary state, and $(m, J)$ labels Ishibashi states, closed string states that can propagate in the transverse channel of an annulus, where $m$ refers to a state in the bulk theory, and $J$ is a degeneracy label. Which $m$ 's appear and with which degeneracy is determined by the modular invariant partition function. Eq. (A.8) must be satisfied for every Ishibashi state which contains massless spinors. ${ }^{12}$ This is determined only by $m$ and not by $J$.

In order to derive (A.6) it turns out that we need only a subset of these conditions namely

$$
\sum_{x} q_{x} N_{x}\left(R_{x(m, J)}-R_{x^{c}(m, J)}\right) w_{m}=0
$$

where $w_{m}$ is the Witten index, counting the difference of spinors and anti-spinors in a character. No sum over $m$ is implied. This condition is a subset of (A.8) because in general there are some Ishibashi states with an equal number of spinors and anti-spinors, which would contribute to U(1) mass, but not to (A.9). Hence the condition we will derive is a necessary, but not sufficient condition for a vanishing $\mathrm{U}(1)$ mass.

We now perform a transformation from the transverse channel to the direct channel of the annulus, in a completely analogous way as the derivation of cubic anomaly cancellation from tadpole cancellation. The Witten indices transform exactly like characters, but are constants. Hence under this transformation we get $w_{m}=\sum_{i} w_{i} S_{i m}$, where $S$ is the modular transformation matrix. Now we multiply the equations with a factor

$$
\sum_{J^{\prime}} \frac{R_{a\left(m, J^{\prime}\right)} g_{J^{\prime} J}^{\Omega, m}}{S_{0 m}}
$$

\footnotetext{
${ }^{12}$ In [48] it is stated erroneously that this condition should hold for all Ishibashi states. However, in the actual standard model search presented in this paper, the condition was limited to Ishibashi states containing massless spinors.
} 
where $g^{\Omega, m}$ is the Ishibashi metric [69] on each degeneracy space. Finally we sum over $m$ and $J$ to obtain

$$
\sum_{x} q_{x} N_{x} \sum_{i} w_{i} \sum_{m, J^{\prime}, J} \frac{S_{i m} R_{a\left(m, J^{\prime}\right)} g_{J^{\prime} J}^{\Omega, m} R_{x(m, J)}}{S_{0 m}}-\left(x \rightarrow x^{c}\right)=0 .
$$

The last sum is precisely the expression for the annulus coefficients, and hence we get

$$
\sum_{x} q_{x} N_{x} \sum_{i} w_{i}\left(A_{a x}^{i}-A_{a x^{c}}^{i}\right)=0
$$

The contraction with the chiral characters $w_{i}$ turns this into the chiral intersection, i.e the first term is precisely $\#\left(\square_{a}, \square_{x}\right)$ as defined above. This expression should hold for any boundary state label $a$. If one chooses a label $a$ that coincides with one of the labels $x$ which participates in the $\mathrm{U}(1)$ symmetry of interests (i.e. $q_{a} N_{a} \neq 0$ ), then one may write

$$
\sum_{i} w^{i} A_{a a}^{i}=\frac{1}{2} \sum_{i} w_{i}\left(A_{a a}^{i}+M_{a}^{i}\right)+\frac{1}{2} \sum_{i} w_{i}\left(A_{a a}^{i}-M_{a}^{i}\right)=\left(\#\left(\square_{a}\right)+\#\left(\Xi_{a}\right)\right)
$$

Note that the sum in (A.8) is over pairs $\left(x, x^{c}\right)$ of conjugate boundary labels, labelled by $x$. Furthermore, if (A.12) holds for a label $a$ it automatically holds for its conjugate $a^{c}$, because $\sum_{i} w_{i} A_{a x}^{i}=-\sum_{i} w_{i} A_{a^{c} x^{c}}$. Hence we can use the same basis of pairs $\left(a, a^{c}\right)$ for all boundary labels, and then only the case $x=a$ can occur. The final result is then indeed precisely (A.6).

Note that using the completeness condition for boundaries [70], $\sum_{a} R^{*} a_{m, J} R^{*} a_{m^{\prime}, J^{\prime}}=$ $\delta_{m, m^{\prime}} \delta_{J, J^{\prime}}$, one can invert the derivation, so that (A.9) can be derived from (A.6). However (A.8) does not follow, and hence, as already stated above, (A.6) is in general only a necessary condition for masslessness of a $\mathrm{U}(1)$. We have examined in a few cases how close it is to being sufficient. It turns out that very often the quantity $R_{x(m, J)}-R_{x^{c}(m, J)}$ vanishes for all $x$ if the Witten index of $m$ is zero. This is true, for example, for all modular invariant partition functions and all orientifolds of the tensor products $(3,3,3,3,3)$, $(3,8,8,8),(6,6,6,6)$ and $(2,2,2,2,2,2)$. Hence in all these cases (A.6) is actually sufficient, provided all boundary labels $a$ are taken into account. The tensor product $(4,4,10,10)$ provides some examples where $R_{x(m, J)}-R_{x^{c}(m, J)}$ does not vanish if $w_{m}=0$, but only for a relatively small set of values of $x$. So cases where (A.6) is not sufficient are rare.

The practical use of (A.6) is in determining if a postulated brane configuration has any chance of having a massless $\mathrm{U}(1)$ boson (for example $Y$ ) in an explicit realization in string theory. Once one has found such a realization, one might as well check (A.8) directly. Hence in practice the set of labels $a$ for which one uses it is just the set of branes appearing in the postulated brane configuration. Then certainly it is just a necessary, and not a sufficient condition.

This condition also plays a rôle in the discussion of charge violation by instantons. Then $a$ is a candidate instanton brane, and the left-hand side of (A.12) must be non-zero to get the required charge violation. Clearly a non-vanishing charge violation implies that the corresponding $\mathrm{U}(1)$ must be massive, but the converse is not necessarily true: for a 
massive $\mathrm{U}(1)$ it may happen that there are no branes that violate conservation of the charge. This was pointed out already in [16] (in particular footnote 16 in that paper). We see now that this can only happen if there are contributions to the vector boson mass from Ishibashi states with a vanishing Witten index.

\section{A.4 Phenomenological requirements}

There are various phenomenological constraints which arise from experiments. We list them below.

- All the Yukawa couplings that give masses to the three families are realized, either perturbatively or non-perturbatively via D-instantons. Thus we require the presence of the terms $10105^{\mathrm{H}}, \mathbf{1 0} \overline{\mathbf{5}} \overline{5}^{\mathrm{H}}$ and $\overline{\mathbf{5}} 5^{\mathrm{H}} \mathbf{1}$.

- For the flipped SU(5) model we require the presence of terms $10^{\mathbf{H}} \mathbf{1 0}^{\mathrm{H}} 5^{\mathrm{H}}$ and $\overline{\mathbf{1 0}}^{\mathbf{H}} \overline{\mathbf{1 0}}^{\mathbf{H}} \overline{\mathbf{5}}^{\mathbf{H}}$ which are crucial for the breaking pattern of the flipped SU(5) down to the standard model gauge symmetry.

- We forbid any R-parity violating couplings $10 \overline{\mathbf{5}} \overline{5}$ or $\overline{\mathbf{5}} 5^{\mathrm{H}}$ on perturbative or nonperturbative level. Specifically that implies that none of the instantons whose presence is required to induce some of the missing but desired couplings induces also the R-parity violating couplings.

- We forbid the presence of the dimension five operator $101010 \overline{5}$ on perturbative or non-perturbative level. For the flipped $\mathrm{SU}(5)$ model we also require the absence of the dimension five operator $\mathbf{1 0} \overline{\mathbf{5}} \overline{\mathbf{5}} \mathbf{1}$, again on perturbative and non-perturbative level. As before that implies that none of the instantons whose presence is required to induce some of the missing but desired couplings induces also these dimension five operators.

- For the Georgi-Glashow D-brane realization often times an instanton which is required to generate a desired Yukawa coupling also induces a tadpole $\mathbf{1}$ and thus an instability for the setup. We rule out any setup which requires the presence of such an instanton.

\section{B Georgi-Glashow realizations based on three stacks}

In this appendix we present all 3-stack realizations of the Georgi-Glashow model that satisfy all the string consistency conditions as well as all the phenomenological constraints laid out in appendix A. We distinguish between two different types of setups, for the first type the $\mu$-term is perturbatively realized and for the second type the $\mu$-term is perturbatively forbidden and must be generated non-perturbatively.

In table 9 we display all possible solutions with exactly three right-handed neutrinos. In the second line we display all possible origins for the matter fields. ${ }^{13}$ We find 12 different

\footnotetext{
${ }^{13}$ Note that this is true up to symmetries. For instance we take into account the symmetry under the exchange of stack $b$ with stack $c$. Moreover here we only display solutions with a perturbatively realized $\mu$-term.
} 


\begin{tabular}{|c|c|c|c|c|c|c|c|c|c|c|c|c|}
\hline \multirow{2}{*}{ Solution \# } & $\mathbf{1 0}$ & $\overline{\mathbf{5}}$ & $\mathbf{5}^{\mathbf{H}}$ & $\overline{\mathbf{5}}^{\mathbf{H}}$ & \multicolumn{7}{|c|}{$\mathbf{| c | c |}$} \\
\cline { 2 - 16 } & $\Xi_{a}$ & $(\bar{a}, b)$ & $(a, \bar{c})$ & $(\bar{a}, c)$ & $(b, \bar{c})$ & $(\bar{b}, c)$ & $(b, c)$ & $(\bar{b}, \bar{c})$ & $\square_{b}$ & $\bar{\square}_{b}$ & $\square_{c}$ & $\bar{\square}_{c}$ \\
\hline 1 & 3 & 3 & 1 & 1 & 0 & 0 & 0 & 0 & 0 & 0 & 0 & 3 \\
\hline 2 & 3 & 3 & 1 & 1 & 0 & 0 & 0 & 1 & 0 & 1 & 0 & 1 \\
\hline 3 & 3 & 3 & 1 & 1 & 1 & 0 & 0 & 0 & 1 & 0 & 0 & 1 \\
\hline $4^{*}$ & 3 & 3 & 1 & 1 & 0 & 0 & 0 & 0 & 0 & 3 & 0 & 0 \\
\hline 5 & 3 & 3 & 1 & 1 & 0 & 0 & 0 & 3 & 0 & 0 & 0 & 0 \\
\hline 6 & 3 & 3 & 1 & 1 & 0 & 3 & 0 & 0 & 0 & 0 & 0 & 0 \\
\hline $7^{*}$ & 3 & 3 & 1 & 1 & 3 & 0 & 0 & 0 & 0 & 0 & 0 & 0 \\
\hline $8^{*}$ & 3 & 3 & 1 & 1 & 0 & 1 & 0 & 1 & 1 & 0 & 0 & 0 \\
\hline $9^{*}$ & 3 & 3 & 1 & 1 & 0 & 0 & 0 & 0 & 3 & 0 & 0 & 0 \\
\hline 10 & 3 & 3 & 1 & 1 & 0 & 1 & 0 & 0 & 0 & 1 & 1 & 0 \\
\hline 11 & 3 & 3 & 1 & 1 & 1 & 0 & 0 & 1 & 0 & 0 & 1 & 0 \\
\hline 12 & 3 & 3 & 1 & 1 & 0 & 0 & 0 & 0 & 0 & 0 & 3 & 0 \\
\hline
\end{tabular}

Table 9. 3-stack quiver realizations of the Georgi-Glashow model with pert. $\mu$-term.

D-brane configurations, where solutions marked with a $*$ potentially exhibit a massless $\mathrm{U}(1)$. In section 2.1.1 we discuss in detail the phenomenology of the configuration 6 .

In table 10 we display all possible 3 D-brane-stack realizations of the Georgi-Glashow model in which the $\mu$-term is not perturbatively realized. These satisfy the severe string consistency constraints as well as the phenomenological conditions laid out in the previous appendix. Again solutions marked with a $*$ potentially exhibit a massless $\mathrm{U}(1)$. In section 2.1.2 we discuss the configuration 9 as a representative with respect to their phenomenology in detail.

\section{Globally consistent 3-stack model}

Here we present globally consistent Gepner configurations that give rise to a GeorgiGlashow-like structure and exhibit an instanton that induces the perturbatively missing Yukawa coupling $10105^{\mathrm{H}}$. We find two different types of global realizations and we present a representative of each here. Before doing so let us however explain the notation in the tables to come.

In the first column the table displays the whole number of states for a particular sector. The last column gives the net chirality of these states. The gauge groups are displayed in the first row, where a $V$ stands for fundamental, $\bar{V}$ for anti-fundamental, $S$ for the symmetric, $A$ for the anti-symmetric and $A d$ for the adjoint under the respective gauge group. The column denoted by Ins represents the rigid $O(1)$-instanton which will induce the Yukawa coupling $1010 \mathbf{5}^{\mathbf{H}}$. Fields charged with respect to it denote the charged zero 


\begin{tabular}{|c|c|c|c|c|c|c|c|c|c|c|c|c|}
\hline \multirow{2}{*}{ Solution \# } & 10 & $\overline{5}$ & $\overline{5}^{\mathrm{H}}$ & $5^{\mathrm{H}}$ & \multicolumn{8}{|c|}{1} \\
\hline & $\theta_{a}$ & $(\bar{a}, b)$ & $(\bar{a}, \bar{b})$ & $(a, c)$ & $(b, \bar{c})$ & $(\bar{b}, c)$ & $(b, c)$ & $(\bar{b}, \bar{c})$ & $\square \square_{b}$ & $\bar{\square}_{b}$ & $\square \square_{c}$ & $\bar{\square}_{c}$ \\
\hline 1 & 3 & 3 & 1 & 1 & 0 & 0 & 0 & 1 & 0 & 0 & 0 & 2 \\
\hline $2^{*}$ & 3 & 3 & 1 & 1 & 0 & 0 & 0 & 0 & 0 & 2 & 0 & 1 \\
\hline 3 & 3 & 3 & 1 & 1 & 1 & 0 & 1 & 0 & 0 & 0 & 0 & 1 \\
\hline $4^{*}$ & 3 & 3 & 1 & 1 & 0 & 0 & 0 & 2 & 0 & 1 & 0 & 0 \\
\hline 5 & 3 & 3 & 1 & 1 & 1 & 0 & 0 & 1 & 1 & 0 & 0 & 0 \\
\hline 6 & 3 & 3 & 1 & 1 & 0 & 0 & 1 & 0 & 2 & 0 & 0 & 0 \\
\hline 7 & 3 & 3 & 1 & 1 & 1 & 0 & 0 & 0 & 0 & 1 & 1 & 0 \\
\hline 8 & 3 & 3 & 1 & 1 & 0 & 0 & 2 & 0 & 0 & 0 & 1 & 0 \\
\hline 9 & 3 & 3 & 1 & 1 & 0 & 0 & 0 & 0 & 1 & 0 & 2 & 0 \\
\hline
\end{tabular}

Table 10. 3-stack quiver realizations of the Georgi-Glashow model with non-pert. $\mu$-term.

modes. One can easily see that the representatives below indeed exhibit the correct charged zero mode structure to induce the coupling $10105^{\mathrm{H}}$.

\section{C.1 Gepner orientifold of type I}

We find 6 global realizations with gauge group $\mathrm{U}(\mathbf{5}) \times \mathrm{U}(\mathbf{1}) \times \mathbf{O}(\mathbf{1}) \times O(2) \times O(1) \times O(1) \times$ $\mathrm{U}(1) \times O(1) \times O(1) \times \mathrm{U}(3)$ where the first 3 gauge groups (the highlighted ones) denote the visible sector. The 6 different global realizations differentiate only in their massless spectrum in the hidden sector, the visible sector is for all 6 realizations the same.

Let us specify the Gepner orientifold. The internal sector of these models consists of a tensor product of four copies of $\mathcal{N}=2$ superconformal minimal models with levels $k_{i}=\{1,10,22,22\}$. This tensor product has 50 symmetric modular invariant partition functions. The one of our interest yields a closed string spectrum characterized by Hodge numbers $h_{11}=32, h_{12}=20$ and 237 singlets. These numbers identify it uniquely. This MIPF allows 4 different orientifold choices, according to the prescription given in [69]. The results below were obtained for one of these four (according to the labelling conventions used in [50] this case corresponds to MIPF nr. 26, orientifold nr. 1). A representative of these 6 realizations is displayed in table 11 .

Here we divided the table into the following segments: the standard model fields (1-5), where the neutrinos arise from the non-chiral sector displayed in line 5 , the instanton zero modes (6-7), chiral observable-hidden matter (8-15), non-chiral observable-hidden matter (16-23), non-chiral observable rank two tensors (24-30), chiral matter within the hidden sector (31-42), and non-chiral matter within the hidden sector (42-53).

Anti-symmetric tensors for $O(1)$ and $\mathrm{U}(1)$ are shown even though their ground state dimension vanishes. The multiplicities of these sectors are however well-defined, and they manifest themselves at higher excitation levels and, if they are chiral, through the tadpole 
cancellation condition (A.2). Note that anti-symmetric tensors are important if a matter brane is converted to an instanton brane, because they lead to additional zero-modes that kill the amplitude. As is clear from the tables, they are completely absent for the instantons we consider.

\section{C.2 Gepner orientifold of type II}

The difference to the six solutions above is that the hidden gauge symmetry is slightly different. The whole gauge symmetry is $\mathrm{U}(\mathbf{5}) \times \mathrm{U}(\mathbf{1}) \times \mathbf{O}(\mathbf{1}) \times \mathrm{U}(1) \times O(2) \times O(1) \times O(2) \times$ $O(1) \times O(1) \times O(1) \times \mathrm{U}(3)$, where again the first three gauge groups denote the visible sector. We find 12 different solutions which have again the same spectrum in the visible sector but different massless spectrum in the hidden sector. The Gepner orientifold is the same as above. Below we display the spectrum of one representative.

Again we divide the table into the different segments: the standard model fields (1-5), where the neutrinos arise from the non-chiral sector displayed in line 5 , the instanton zero modes (6-7), chiral observable-hidden matter (8-16), non-chiral observable-hidden matter (17-24), non-chiral observable rank two tensors (25-31), chiral matter within the hidden sector (32-41), and non-chiral matter within the hidden sector (42-59). 


\begin{tabular}{|c|c|c|c|c|c|c|c|c|c|c|c|c|c|}
\hline Num. & Mult. & $\mathrm{U}(5)$ & $\mathrm{U}(1)$ & $\mathrm{O}(1)$ & $\mathrm{O}(2)$ & $\mathrm{O}(1)$ & $\mathrm{O}(1)$ & $\mathrm{U}(1)$ & $\mathrm{O}(1)$ & $\mathrm{O}(1)$ & $\mathrm{U}(3)$ & Inst & Chir. \\
\hline 1 & 3 & $\mathrm{~A}$ & 0 & 0 & 0 & 0 & 0 & 0 & 0 & 0 & 0 & 0 & 3 \\
\hline 2 & 3 & V & $\mathrm{V}^{*}$ & 0 & 0 & 0 & 0 & 0 & 0 & 0 & 0 & 0 & -3 \\
\hline 3 & 3 & V & V & 0 & 0 & 0 & 0 & 0 & 0 & 0 & 0 & 0 & -3 \\
\hline 4 & 3 & V & 0 & V & 0 & 0 & 0 & 0 & 0 & 0 & 0 & 0 & 3 \\
\hline 5 & 12 & 0 & V & V & 0 & 0 & 0 & 0 & 0 & 0 & 0 & 0 & 0 \\
\hline 6 & 1 & $\mathrm{~V}$ & 0 & 0 & 0 & 0 & 0 & 0 & 0 & 0 & 0 & $\mathrm{~V}$ & -1 \\
\hline 7 & 1 & 0 & 0 & V & 0 & 0 & 0 & 0 & 0 & 0 & 0 & V & 0 \\
\hline 8 & 1 & $\overline{\mathrm{V}}$ & 0 & 0 & $\overline{\mathrm{V}}$ & 0 & 0 & 0 & 0 & 0 & 0 & 0 & 1 \\
\hline 9 & 1 & V & 0 & 0 & 0 & 0 & 0 & $\mathrm{~V}^{*}$ & 0 & 0 & 0 & 0 & 1 \\
\hline 10 & 1 & V & 0 & 0 & 0 & 0 & 0 & 0 & V & 0 & 0 & 0 & -1 \\
\hline 11 & 1 & V & 0 & 0 & 0 & 0 & 0 & 0 & 0 & V & 0 & 0 & 1 \\
\hline 12 & 1 & V & 0 & 0 & 0 & 0 & 0 & 0 & 0 & 0 & $\mathrm{~V}^{*}$ & 0 & -1 \\
\hline 13 & 1 & 0 & V & 0 & 0 & 0 & 0 & $\mathrm{~V}^{*}$ & 0 & 0 & 0 & 0 & 1 \\
\hline 14 & 1 & 0 & V & 0 & 0 & 0 & 0 & V & 0 & 0 & 0 & 0 & -1 \\
\hline 15 & 1 & 0 & 0 & V & 0 & 0 & 0 & 0 & 0 & 0 & V & 0 & -1 \\
\hline 16 & 2 & V & 0 & 0 & 0 & 0 & 0 & V & 0 & 0 & 0 & 0 & 0 \\
\hline 17 & 4 & V & 0 & 0 & 0 & 0 & 0 & 0 & 0 & 0 & $\mathrm{~V}$ & 0 & 0 \\
\hline 18 & 4 & 0 & V & 0 & V & 0 & 0 & 0 & 0 & 0 & 0 & 0 & 0 \\
\hline 19 & 6 & 0 & V & 0 & 0 & $\mathrm{~V}$ & 0 & 0 & 0 & 0 & 0 & 0 & 0 \\
\hline 20 & 2 & 0 & V & 0 & 0 & 0 & V & 0 & 0 & 0 & 0 & 0 & 0 \\
\hline 21 & 2 & 0 & 0 & V & V & 0 & 0 & 0 & 0 & 0 & 0 & 0 & 0 \\
\hline 22 & 2 & 0 & 0 & V & 0 & $\mathrm{~V}$ & 0 & 0 & 0 & 0 & 0 & 0 & 0 \\
\hline 23 & 4 & 0 & 0 & V & 0 & 0 & 0 & 0 & $\mathrm{~V}$ & 0 & 0 & 0 & 0 \\
\hline 24 & 3 & $\mathrm{Ad}$ & 0 & 0 & 0 & 0 & 0 & 0 & 0 & 0 & 0 & 0 & 0 \\
\hline 25 & 2 & $\mathrm{~S}$ & 0 & 0 & 0 & 0 & 0 & 0 & 0 & 0 & 0 & 0 & 0 \\
\hline 26 & 7 & 0 & $\mathrm{Ad}$ & 0 & 0 & 0 & 0 & 0 & 0 & 0 & 0 & 0 & 0 \\
\hline 27 & 8 & 0 & $\mathrm{~S}$ & 0 & 0 & 0 & 0 & 0 & 0 & 0 & 0 & 0 & 0 \\
\hline 28 & 8 & 0 & A & 0 & 0 & 0 & 0 & 0 & 0 & 0 & 0 & 0 & 0 \\
\hline 29 & 5 & 0 & 0 & A & 0 & 0 & 0 & 0 & 0 & 0 & 0 & 0 & 0 \\
\hline 30 & 4 & 0 & 0 & $\mathrm{~S}$ & 0 & 0 & 0 & 0 & 0 & 0 & 0 & 0 & 0 \\
\hline 31 & 1 & 0 & 0 & 0 & $\bar{V}$ & 0 & 0 & $\mathrm{~V}$ & 0 & 0 & 0 & 0 & 1 \\
\hline 32 & 1 & 0 & 0 & 0 & 0 & V & 0 & $\mathrm{~V}$ & 0 & 0 & 0 & 0 & -1 \\
\hline 33 & 1 & 0 & 0 & 0 & 0 & 0 & $\mathrm{~V}$ & $\mathrm{~V}$ & 0 & 0 & 0 & 0 & -1 \\
\hline 34 & 1 & 0 & 0 & 0 & 0 & 0 & 0 & $\mathrm{~S}$ & 0 & 0 & 0 & 0 & 1 \\
\hline 35 & 1 & 0 & 0 & 0 & 0 & 0 & 0 & A & 0 & 0 & 0 & 0 & -1 \\
\hline 36 & 1 & 0 & 0 & 0 & 0 & 0 & 0 & $\mathrm{~V}$ & $\mathrm{~V}$ & 0 & 0 & 0 & 1 \\
\hline 37 & 1 & 0 & 0 & 0 & 0 & 0 & 0 & $\mathrm{~V}$ & 0 & $\mathrm{~V}$ & 0 & 0 & 1 \\
\hline 38 & 1 & 0 & 0 & 0 & 0 & 0 & 0 & $\mathrm{~V}$ & 0 & 0 & $\mathrm{~V}$ & 0 & -1 \\
\hline 39 & 1 & 0 & 0 & 0 & 0 & 0 & 0 & 0 & V & 0 & V & 0 & 1 \\
\hline
\end{tabular}

Table 11: Complete spectrum of a global model of type I. (Continued on next page) 
Table 11 (continued from previous page)

\begin{tabular}{|c|c|c|c|c|c|c|c|c|c|c|c|c|c|}
\hline Num. & Mult. & $\mathrm{U}(5)$ & $\mathrm{U}(1)$ & $\mathrm{O}(1)$ & $\mathrm{O}(2)$ & $\mathrm{O}(1)$ & $\mathrm{O}(1)$ & $\mathrm{U}(1)$ & $\mathrm{O}(1)$ & $\mathrm{O}(1)$ & $\mathrm{U}(3)$ & Inst & Chir. \\
\hline 40 & 2 & 0 & 0 & 0 & 0 & 0 & 0 & 0 & 0 & $\mathrm{~V}$ & $\mathrm{~V}$ & 0 & 2 \\
41 & 1 & 0 & 0 & 0 & 0 & 0 & 0 & 0 & 0 & 0 & $\mathrm{~S}$ & 0 & -1 \\
42 & 1 & 0 & 0 & 0 & 0 & 0 & 0 & 0 & 0 & 0 & $\mathrm{~A}$ & 0 & -1 \\
\hline 43 & 1 & 0 & 0 & 0 & $\mathrm{~S}$ & 0 & 0 & 0 & 0 & 0 & 0 & 0 & 0 \\
44 & 1 & 0 & 0 & 0 & $\mathrm{~V}$ & $\mathrm{~V}$ & 0 & 0 & 0 & 0 & 0 & 0 & 0 \\
45 & 1 & 0 & 0 & 0 & 0 & $\mathrm{~V}$ & $\mathrm{~V}$ & 0 & 0 & 0 & 0 & 0 & 0 \\
46 & 1 & 0 & 0 & 0 & 0 & $\mathrm{~S}$ & 0 & 0 & 0 & 0 & 0 & 0 & 0 \\
47 & 1 & 0 & 0 & 0 & 0 & $\mathrm{~V}$ & $\mathrm{~V}$ & 0 & 0 & 0 & 0 & 0 & 0 \\
48 & 2 & 0 & 0 & 0 & 0 & $\mathrm{~V}$ & 0 & 0 & $\mathrm{~V}$ & 0 & 0 & 0 & 0 \\
49 & 2 & 0 & 0 & 0 & 0 & $\mathrm{~V}$ & 0 & 0 & 0 & $\mathrm{~V}$ & 0 & 0 & 0 \\
50 & 1 & 0 & 0 & 0 & 0 & 0 & $\mathrm{~S}$ & 0 & 0 & 0 & 0 & 0 & 0 \\
51 & 1 & 0 & 0 & 0 & 0 & 0 & $\mathrm{~V}$ & 0 & $\mathrm{~V}$ & 0 & 0 & 0 & 0 \\
52 & 1 & 0 & 0 & 0 & 0 & 0 & $\mathrm{~V}$ & 0 & 0 & $\mathrm{~V}$ & 0 & 0 & 0 \\
53 & 1 & 0 & 0 & 0 & 0 & 0 & 0 & $\mathrm{Ad}$ & 0 & 0 & 0 & 0 & 0 \\
54 & 1 & 0 & 0 & 0 & 0 & 0 & 0 & 0 & $\mathrm{~A}$ & 0 & 0 & 0 & 0 \\
\hline
\end{tabular}




\begin{tabular}{|c|c|c|c|c|c|c|c|c|c|c|c|c|c|c|}
\hline Num. & Mult. & $\mathrm{U}(5)$ & $\mathrm{U}(1)$ & $\mathrm{O}(1)$ & $\mathrm{U}(1)$ & $\mathrm{O}(2)$ & $\mathrm{O}(1)$ & $\mathrm{O}(2)$ & $\mathrm{O}(1)$ & $\mathrm{O}(1)$ & $\mathrm{O}(1)$ & $\mathrm{U}(3)$ & Inst. & Chir. \\
\hline 1 & 3 & A & 0 & 0 & 0 & 0 & 0 & 0 & 0 & 0 & 0 & 0 & 0 & 3 \\
\hline 2 & 3 & V & $\mathrm{V}^{*}$ & 0 & 0 & 0 & 0 & 0 & 0 & 0 & 0 & 0 & 0 & -3 \\
\hline 3 & 3 & V & V & 0 & 0 & 0 & 0 & 0 & 0 & 0 & 0 & 0 & 0 & -3 \\
\hline 4 & 3 & V & 0 & V & 0 & 0 & 0 & 0 & 0 & 0 & 0 & 0 & 0 & 3 \\
\hline 5 & 6 & 0 & V & V & 0 & 0 & 0 & 0 & 0 & 0 & 0 & 0 & 0 & 0 \\
\hline 6 & 1 & V & 0 & 0 & 0 & 0 & 0 & 0 & 0 & 0 & 0 & 0 & V & -1 \\
\hline 7 & 1 & 0 & 0 & V & 0 & 0 & 0 & 0 & 0 & 0 & 0 & 0 & V & 0 \\
\hline 8 & 1 & $\overline{\mathrm{V}}$ & 0 & 0 & 0 & $\bar{V}$ & 0 & 0 & 0 & 0 & 0 & 0 & 0 & 1 \\
\hline 9 & 1 & V & 0 & 0 & 0 & 0 & 0 & V & 0 & 0 & 0 & 0 & 0 & 1 \\
\hline 10 & 1 & V & 0 & 0 & 0 & 0 & 0 & 0 & V & 0 & 0 & 0 & 0 & -1 \\
\hline 11 & 1 & V & 0 & 0 & 0 & 0 & 0 & 0 & 0 & V & 0 & 0 & 0 & -1 \\
\hline 12 & 1 & V & 0 & 0 & 0 & 0 & 0 & 0 & 0 & 0 & V & 0 & 0 & 1 \\
\hline 13 & 1 & V & 0 & 0 & 0 & 0 & 0 & 0 & 0 & 0 & 0 & $\mathrm{~V}^{*}$ & 0 & -1 \\
\hline 14 & 1 & 0 & V & 0 & V & 0 & 0 & 0 & 0 & 0 & 0 & 0 & 0 & 1 \\
\hline 15 & 1 & 0 & V & 0 & $\mathrm{~V}^{*}$ & 0 & 0 & 0 & 0 & 0 & 0 & 0 & 0 & -1 \\
\hline 16 & 1 & 0 & 0 & V & V & 0 & 0 & 0 & 0 & 0 & 0 & 0 & 0 & 1 \\
\hline 17 & 4 & V & 0 & 0 & 0 & 0 & 0 & 0 & 0 & 0 & 0 & V & 0 & 0 \\
\hline 18 & 4 & 0 & V & 0 & 0 & V & 0 & 0 & 0 & 0 & 0 & 0 & 0 & 0 \\
\hline 19 & 2 & 0 & V & 0 & 0 & 0 & V & 0 & 0 & 0 & 0 & 0 & 0 & 0 \\
\hline 20 & 6 & 0 & V & 0 & 0 & 0 & 0 & V & 0 & 0 & 0 & 0 & 0 & 0 \\
\hline 21 & 1 & 0 & 0 & V & 0 & V & 0 & 0 & 0 & 0 & 0 & 0 & 0 & 0 \\
\hline 22 & 1 & 0 & 0 & V & 0 & 0 & 0 & V & 0 & 0 & 0 & 0 & 0 & 0 \\
\hline 23 & 3 & 0 & 0 & V & 0 & 0 & 0 & 0 & V & 0 & 0 & 0 & 0 & 0 \\
\hline 24 & 4 & 0 & 0 & V & 0 & 0 & 0 & 0 & 0 & V & 0 & 0 & 0 & 0 \\
\hline 25 & 3 & $\mathrm{Ad}$ & 0 & 0 & 0 & 0 & 0 & 0 & 0 & 0 & 0 & 0 & 0 & 0 \\
\hline 26 & 2 & $\mathrm{~S}$ & 0 & 0 & 0 & 0 & 0 & 0 & 0 & 0 & 0 & 0 & 0 & 0 \\
\hline 27 & 7 & 0 & $\mathrm{Ad}$ & 0 & 0 & 0 & 0 & 0 & 0 & 0 & 0 & 0 & 0 & 0 \\
\hline 28 & 8 & 0 & $\mathrm{~S}$ & 0 & 0 & 0 & 0 & 0 & 0 & 0 & 0 & 0 & 0 & 0 \\
\hline 29 & 8 & 0 & A & 0 & 0 & 0 & 0 & 0 & 0 & 0 & 0 & 0 & 0 & 0 \\
\hline 30 & 1 & 0 & 0 & $\mathrm{~S}$ & 0 & 0 & 0 & 0 & 0 & 0 & 0 & 0 & 0 & 0 \\
\hline 31 & 1 & 0 & 0 & A & 0 & 0 & 0 & 0 & 0 & 0 & 0 & 0 & 0 & 0 \\
\hline 32 & 1 & 0 & 0 & 0 & $\bar{S}$ & 0 & 0 & 0 & 0 & 0 & 0 & 0 & 0 & -1 \\
\hline 33 & 1 & 0 & 0 & 0 & A & 0 & 0 & 0 & 0 & 0 & 0 & 0 & 0 & 1 \\
\hline 34 & 1 & 0 & 0 & 0 & V & V & 0 & 0 & 0 & 0 & 0 & 0 & 0 & 1 \\
\hline 35 & 1 & 0 & 0 & 0 & V & 0 & V & 0 & 0 & 0 & 0 & 0 & 0 & 1 \\
\hline 36 & 1 & 0 & 0 & 0 & V & 0 & 0 & V & 0 & 0 & 0 & 0 & 0 & 1 \\
\hline 37 & 1 & 0 & 0 & 0 & 0 & 0 & 0 & V & 0 & 0 & 0 & V & 0 & -1 \\
\hline 38 & 1 & 0 & 0 & 0 & 0 & 0 & 0 & 0 & V & 0 & 0 & V & 0 & 1 \\
\hline 39 & 2 & 0 & 0 & 0 & 0 & 0 & 0 & 0 & 0 & 0 & V & V & 0 & 2 \\
\hline
\end{tabular}

Table 12: Complete spectrum of a global model of type II. (Continued on next page) 
Table 12 (continued from previous page)

\begin{tabular}{|c|c|c|c|c|c|c|c|c|c|c|c|c|c|c|}
\hline Num. & Mult. & $\mathrm{U}(5)$ & $\mathrm{U}(1)$ & $\mathrm{O}(1)$ & $\mathrm{U}(1)$ & $\mathrm{O}(2)$ & $\mathrm{O}(1)$ & $\mathrm{O}(2)$ & $\mathrm{O}(1)$ & $\mathrm{O}(1)$ & $\mathrm{O}(1)$ & $\mathrm{U}(3)$ & Inst. & Chir. \\
\hline 40 & 1 & 0 & 0 & 0 & 0 & 0 & 0 & 0 & 0 & 0 & 0 & $\mathrm{~S}$ & 0 & -1 \\
41 & 1 & 0 & 0 & 0 & 0 & 0 & 0 & 0 & 0 & 0 & 0 & $\mathrm{~A}$ & 0 & -1 \\
\hline 42 & 1 & 0 & 0 & 0 & $\mathrm{Ad}$ & 0 & 0 & 0 & 0 & 0 & 0 & 0 & 0 & 0 \\
43 & 2 & 0 & 0 & 0 & $\mathrm{~V}$ & 0 & 0 & 0 & 0 & 0 & 0 & $\mathrm{~V}^{*}$ & 0 & 0 \\
44 & 1 & 0 & 0 & 0 & 0 & $\mathrm{~S}$ & 0 & 0 & 0 & 0 & 0 & 0 & 0 & 0 \\
45 & 1 & 0 & 0 & 0 & 0 & $\mathrm{~V}$ & $\mathrm{~V}$ & 0 & 0 & 0 & 0 & 0 & 0 & 0 \\
46 & 1 & 0 & 0 & 0 & 0 & $\mathrm{~V}$ & 0 & $\mathrm{~V}$ & 0 & 0 & 0 & 0 & 0 & 0 \\
47 & 1 & 0 & 0 & 0 & 0 & 0 & $\mathrm{~S}$ & 0 & 0 & 0 & 0 & 0 & 0 & 0 \\
48 & 1 & 0 & 0 & 0 & 0 & 0 & $\mathrm{~V}$ & $\mathrm{~V}$ & 0 & 0 & 0 & 0 & 0 & 0 \\
49 & 1 & 0 & 0 & 0 & 0 & 0 & $\mathrm{~V}$ & 0 & $\mathrm{~V}$ & 0 & 0 & 0 & 0 & 0 \\
50 & 1 & 0 & 0 & 0 & 0 & 0 & $\mathrm{~V}$ & 0 & 0 & $\mathrm{~V}$ & 0 & 0 & 0 & 0 \\
51 & 1 & 0 & 0 & 0 & 0 & 0 & $\mathrm{~V}$ & 0 & 0 & 0 & $\mathrm{~V}$ & 0 & 0 & 0 \\
52 & 1 & 0 & 0 & 0 & 0 & 0 & 0 & $\mathrm{~S}$ & 0 & 0 & 0 & 0 & 0 & 0 \\
53 & 3 & 0 & 0 & 0 & 0 & 0 & 0 & $\mathrm{~V}$ & $\mathrm{~V}$ & 0 & 0 & 0 & 0 & 0 \\
54 & 3 & 0 & 0 & 0 & 0 & 0 & 0 & $\mathrm{~V}$ & 0 & $\mathrm{~V}$ & 0 & 0 & 0 & 0 \\
55 & 1 & 0 & 0 & 0 & 0 & 0 & 0 & $\mathrm{~V}$ & 0 & 0 & $\mathrm{~V}$ & 0 & 0 & 0 \\
56 & 1 & 0 & 0 & 0 & 0 & 0 & 0 & 0 & $\mathrm{~A}$ & 0 & 0 & 0 & 0 & 0 \\
57 & 2 & 0 & 0 & 0 & 0 & 0 & 0 & 0 & $\mathrm{~V}$ & $\mathrm{~V}$ & 0 & 0 & 0 & 0 \\
58 & 2 & 0 & 0 & 0 & 0 & 0 & 0 & 0 & 0 & $\mathrm{~A}$ & 0 & 0 & 0 & 0 \\
59 & 1 & 0 & 0 & 0 & 0 & 0 & 0 & 0 & 0 & $\mathrm{~V}$ & $\mathrm{~V}$ & 0 & 0 & 0 \\
\hline
\end{tabular}


Open Access. This article is distributed under the terms of the Creative Commons Attribution Noncommercial License which permits any noncommercial use, distribution, and reproduction in any medium, provided the original author(s) and source are credited.

\section{References}

[1] E. Kiritsis, M. Lennek and B. Schellekens, SU(5) orientifolds, Yukawa couplings, stringy instantons and proton decay, Nucl. Phys. B 829 (2010) 298 [arXiv:0909. 0271] [SPIRES].

[2] R. Blumenhagen, M. Cvetič, P. Langacker and G. Shiu, Toward realistic intersecting D-brane models, Ann. Rev. Nucl. Part. Sci. 55 (2005) 71 [hep-th/0502005] [SPIRES].

[3] R. Blumenhagen, B. Körs, D. Lüst and S. Stieberger, Four-dimensional string compactifications with D-branes, orientifolds and fluxes, Phys. Rept. 445 (2007) 1 [hep-th/0610327] [SPIRES].

[4] F. Marchesano, Progress in D-brane model building, Fortsch. Phys. 55 (2007) 491 [hep-th/0702094] [SPIRES].

[5] M. Cvetič, I. Papadimitriou and G. Shiu, Supersymmetric three family SU(5) grand unified models from type IIA orientifolds with intersecting D6-branes, Nucl. Phys. B 659 (2003) 193 [hep-th/0212177] [SPIRES].

[6] C.M. Chen, G.V. Kraniotis, V.E. Mayes, D.V. Nanopoulos and J.W. Walker, A supersymmetric flipped SU(5) intersecting brane world, Phys. Lett. B 611 (2005) 156 [hep-th/0501182] [SPIRES].

[7] C.M. Chen, G.V. Kraniotis, V.E. Mayes, D.V. Nanopoulos and J.W. Walker, A k-theory anomaly free supersymmetric flipped $\mathrm{SU}(5)$ model from intersecting branes, Phys. Lett. B 625 (2005) 96 [hep-th/0507232] [SPIRES].

[8] C.-M. Chen, V.E. Mayes and D.V. Nanopoulos, Flipped SU(5) from D-branes with type IIB fluxes, Phys. Lett. B 633 (2006) 618 [hep-th/0511135] [SPIRES].

[9] F. Gmeiner and M. Stein, Statistics of SU(5) D-brane models on a type-II orientifold, Phys. Rev. D 73 (2006) 126008 [hep-th/0603019] [SPIRES].

[10] E. Floratos and C. Kokorelis, MSSM GUT string vacua, split supersymmetry and fluxes, hep-th/0607217 [SPIRES].

[11] M. Cvetič and P. Langacker, New grand unified models with intersecting D6-branes, neutrino masses and flipped SU(5), Nucl. Phys. B 776 (2007) 118 [hep-th/0607238] [SPIRES].

[12] I. Antoniadis, A. Kumar and B. Panda, Supersymmetric SU(5) GUT with Stabilized Moduli, Nucl. Phys. B 795 (2008) 69 [arXiv:0709.2799] [SPIRES].

[13] R. Blumenhagen, V. Braun, T.W. Grimm and T. Weigand, GUTs in type IIB orientifold compactifications, Nucl. Phys. B $\mathbf{8 1 5}$ (2009) 1 [arXiv:0811.2936] [SPIRES].

[14] D. Berenstein, Branes vs. GUTS: challenges for string inspired phenomenology, hep-th/0603103 [SPIRES].

[15] R. Blumenhagen, M. Cvetič and T. Weigand, Spacetime instanton corrections in $4 D$ string vacua - The seesaw mechanism for D-brane models, Nucl. Phys. B 771 (2007) 113 [hep-th/0609191] [SPIRES].

[16] L.E. Ibáñez and A.M. Uranga, Neutrino Majorana masses from string theory instanton effects, JHEP 03 (2007) 052 [hep-th/0609213] [SPIRES]. 
[17] B. Florea, S. Kachru, J. McGreevy and N. Saulina, Stringy instantons and quiver gauge theories, JHEP 05 (2007) 024 [hep-th/0610003] [SPIRES].

[18] R. Blumenhagen, M. Cvetič, S. Kachru and T. Weigand, D-brane instantons in type II orientifolds, Ann. Rev. Nucl. Part. Sci. 59 (2009) 269 [arXiv:0902.3251] [SPIRES].

[19] M. Bianchi and M. Samsonyan, Notes on unoriented D-brane instantons, Int. J. Mod. Phys. A 24S1 (2009) 5737 [arXiv:0909.2173] [SPIRES].

[20] R. Argurio, M. Bertolini, S. Franco and S. Kachru, Metastable vacua and D-branes at the conifold, JHEP 06 (2007) 017 [hep-th/0703236] [SPIRES].

[21] R. Argurio, M. Bertolini, G. Ferretti, A. Lerda and C. Petersson, Stringy instantons at orbifold singularities, JHEP 06 (2007) 067 [arXiv: 0704.0262] [SPIRES].

[22] M. Bianchi, F. Fucito and J.F. Morales, D-brane instantons on the $T^{6} / Z_{3}$ orientifold, JHEP 07 (2007) 038 [arXiv: 0704.0784] [SPIRES].

[23] L.E. Ibáñez, A.N. Schellekens and A.M. Uranga, Instanton induced neutrino majorana masses in CFT orientifolds with MSSM-like spectra, JHEP 06 (2007) 011 [arXiv:0704.1079] [SPIRES].

[24] R. Blumenhagen, M. Cvetič, R. Richter, 2 and T. Weigand, LiftiNg D-instanton zero modes by recombination and background fluxes, JHEP 10 (2007) 098 [arXiv:0708.0403] [SPIRES].

[25] I. Garcia-Etxebarria and A.M. Uranga, Non-perturbative superpotentials across lines of marginal stability, JHEP 01 (2008) 033 [arXiv:0711.1430] [SPIRES].

[26] M. Cvetič, R. Richter, 2 and T. Weigand, (Non-)BPS bound states and D-brane instantons, JHEP 07 (2008) 012 [arXiv:0803.2513] [SPIRES].

[27] I. Garcia-Etxebarria, F. Marchesano and A.M. Uranga, Non-perturbative F-terms across lines of BPS stability, JHEP 07 (2008) 028 [arXiv: 0805.0713] [SPIRES].

[28] M. Aganagic, C. Beem and S. Kachru, Geometric transitions and dynamical SUSY breaking, Nucl. Phys. B 796 (2008) 1 [arXiv:0709.4277] [SPIRES].

[29] C. Petersson, Superpotentials from stringy instantons without orientifolds, JHEP 05 (2008) 078 [arXiv:0711.1837] [SPIRES].

[30] I. Garcia-Etxebarria, D-brane instantons and matrix models, JHEP 07 (2009) 017 [arXiv:0810.1482] [SPIRES].

[31] G. Ferretti and C. Petersson, Non-perturbative effects on a fractional D3-brane, JHEP 03 (2009) 040 [arXiv:0901.1182] [SPIRES].

[32] M. Cvetič, J. Halverson and R. Richter, Mass hierarchies from MSSM orientifold compactifications, JHEP 07 (2010) 005 [arXiv: 0909.4292] [SPIRES].

[33] R. Blumenhagen, M. Cvetič, D. Lüst, R. Richter, 2 and T. Weigand, Non-perturbative Yukawa couplings from string instantons, Phys. Rev. Lett. 100 (2008) 061602 [arXiv:0707.1871] [SPIRES].

[34] M. Cvetič, R. Richter, 2 and T. Weigand, Computation of D-brane instanton induced superpotential couplings - Majorana masses from string theory, Phys. Rev. D 76 (2007) 086002 [hep-th/0703028] [SPIRES].

[35] S. Antusch, L.E. Ibáñez and T. Macri, Neutrino masses and mixings from string theory instantons, JHEP 09 (2007) 087 [arXiv:0706.2132] [SPIRES].

[36] M. Cvetič and T. Weigand, Hierarchies from D-brane instantons in globally defined Calabi-Yau orientifolds, Phys. Rev. Lett. 100 (2008) 251601 [arXiv:0711.0209] [SPIRES]. 
[37] L.E. Ibáñez and R. Richter, Stringy instantons and Yukawa couplings in MSSM-like orientifold models, JHEP 03 (2009) 090 [arXiv:0811.1583] [SPIRES].

[38] M. Cvetič and P. Langacker, D-instanton generated Dirac neutrino masses, Phys. Rev. D 78 (2008) 066012 [arXiv:0803.2876] [SPIRES].

[39] C. Angelantonj, M. Bianchi, G. Pradisi, A. Sagnotti and Y.S. Stanev, Comments on Gepner models and type-I vacua in string theory, Phys. Lett. B 387 (1996) 743 [hep-th/9607229] [SPIRES].

[40] R. Blumenhagen and A. Wisskirchen, Spectra of $4 D, N=1$ type-I string vacua on non-toroidal CY threefolds, Phys. Lett. B 438 (1998) 52 [hep-th/9806131] [SPIRES].

[41] R. Blumenhagen, Supersymmetric orientifolds of Gepner models, JHEP 11 (2003) 055 [hep-th/0310244] [SPIRES].

[42] R. Blumenhagen and T. Weigand, Chiral supersymmetric Gepner model orientifolds, JHEP 02 (2004) 041 [hep-th/0401148] [SPIRES].

[43] R. Blumenhagen and T. Weigand, A note on partition functions of Gepner model orientifolds, Phys. Lett. B 591 (2004) 161 [hep-th/0403299] [SPIRES].

[44] R. Blumenhagen and T. Weigand, Chiral Gepner model orientifolds, hep-th/0408147 [SPIRES].

[45] D. Gepner, Exactly solvable string compactifications on manifolds of $\mathrm{SU}(N)$ holonomy, Phys. Lett. B 199 (1987) 380 [SPIRES].

[46] D. Gepner, Space-time supersymmetry in compactified string theory and superconformal models, Nucl. Phys. B 296 (1988) 757 [SPIRES].

[47] T. Eguchi, H. Ooguri, A. Taormina and S.-K. Yang, Superconformal algebras and string compactification on manifolds with $\mathrm{SU}(N)$ holonomy, Nucl. Phys. B 315 (1989) 193 [SPIRES].

[48] T.P.T. Dijkstra, L.R. Huiszoon and A.N. Schellekens, Chiral supersymmetric standard model spectra from orientifolds of Gepner models, Phys. Lett. B 609 (2005) 408 [hep-th/0403196] [SPIRES].

[49] T.P.T. Dijkstra, L.R. Huiszoon and A.N. Schellekens, Supersymmetric standard model spectra from RCFT orientifolds, Nucl. Phys. B 710 (2005) 3 [hep-th/0411129] [SPIRES].

[50] P. Anastasopoulos, T.P.T. Dijkstra, E. Kiritsis and A.N. Schellekens, Orientifolds, hypercharge embeddings and the standard model, Nucl. Phys. B 759 (2006) 83 [hep-th/0605226] [SPIRES].

[51] E. Kiritsis, B. Schellekens and M. Tsulaia, Discriminating MSSM families in (free-field) Gepner orientifolds, JHEP 10 (2008) 106 [arXiv:0809.0083] [SPIRES].

[52] I. Antoniadis, J.R. Ellis, J.S. Hagelin and D.V. Nanopoulos, Supersymmetric flipped SU(5) revitalized, Phys. Lett. B 194 (1987) 231 [SPIRES].

[53] M. Cvetič, J. Halverson and R. Richter, 2, Realistic Yukawa structures from orientifold compactifications, JHEP 12 (2009) 063 [arXiv:0905.3379] [SPIRES].

[54] M. Bianchi and J.F. Morales, Anomalies and tadpoles, JHEP 03 (2000) 030 [hep-th/0002149] [SPIRES].

[55] G.K. Leontaris, Instanton induced charged fermion and neutrino masses in a minimal standard model scenario from intersecting D-branes, Int. J. Mod. Phys. A 24 (2009) 6035 [arXiv: 0903.3691] [SPIRES]. 
[56] P. Anastasopoulos, E. Kiritsis and A. Lionetto, On mass hierarchies in orientifold vacua, JHEP 08 (2009) 026 [arXiv:0905.3044] [SPIRES].

[57] M. Cvetič, J. Halverson, P. Langacker and R. Richter, The Weinberg operator and a lower string scale in orientifold compactifications, JHEP 10 (2010) 094 [arXiv:1001.3148] [SPIRES].

[58] P. Anastasopoulos, G.K. Leontaris and N.D. Vlachos, Phenomenological analysis of D-brane Pati-Salam vacua, JHEP 05 (2010) 011 [arXiv: 1002.2937] [SPIRES].

[59] M. Cvetič, J. Halverson and P. Langacker, Singlet extensions of the MSSM in the quiver landscape, JHEP 09 (2010) 076 [arXiv: 1006.3341] [SPIRES].

[60] R. Blumenhagen, A. Deser and D. Lüst, FCNC processes from D-brane instantons, arXiv: 1007.4770 [SPIRES].

[61] F. Fucito, A. Lionetto, J.F. Morales and R. Richter, Dynamical supersymmetry breaking in intersecting brane models, JHEP 11 (2010) 024 [arXiv: 1007.5449] [SPIRES].

[62] I. Antoniadis, E. Kiritsis and T.N. Tomaras, A D-brane alternative to unification, Phys. Lett. B 486 (2000) 186 [hep-ph/0004214] [SPIRES].

[63] G. Aldazabal, L.E. Ibáñez, F. Quevedo and A.M. Uranga, D-branes at singularities: a bottom-up approach to the string embedding of the standard model, JHEP 08 (2000) 002 [hep-th/0005067] [SPIRES].

[64] I. Antoniadis, E. Kiritsis and T. Tomaras, D-brane standard model, Fortsch. Phys. 49 (2001) 573 [hep-th/0111269] [SPIRES].

[65] G. Aldazabal, S. Franco, L.E. Ibáñez, R. Rabadán and A.M. Uranga, Intersecting brane worlds, JHEP 02 (2001) 047 [hep-ph/0011132] [SPIRES].

[66] I. Antoniadis, E. Kiritsis and J. Rizos, Anomalous U(1)'s in type-I superstring vacua, Nucl. Phys. B 637 (2002) 92 [hep-th/0204153] [SPIRES].

[67] P. Anastasopoulos, $4 D$ anomalous $\mathrm{U}(1)$ 's, their masses and their relation to $6 D$ anomalies, JHEP 08 (2003) 005 [hep-th/0306042] [SPIRES].

[68] P. Anastasopoulos, Anomalous U(1)'s masses in non-supersymmetric open string vacua, Phys. Lett. B 588 (2004) 119 [hep-th/0402105] [SPIRES].

[69] J. Fuchs, L.R. Huiszoon, A.N. Schellekens, C. Schweigert and J. Walcher, Boundaries, crosscaps and simple currents, Phys. Lett. B 495 (2000) 427 [hep-th/0007174] [SPIRES].

[70] G. Pradisi, A. Sagnotti and Y.S. Stanev, Completeness conditions for boundary operators in $2 D$ conformal field theory, Phys. Lett. B 381 (1996) 97 [hep-th/9603097] [SPIRES]. 